\title{
Aziridines versus Vinyl Carbamates from the Direct Amination of Electron Withdrawing Group-Substituted Trifluoromethyl Enoates
}

Stefania Fioravanti, * Daniele Colantoni, Lucio Pellacani, * and Paolo A. Tardella*

Dipartimento di Chimica, Università degli Studi "La Sapienza", P.le Aldo Moro 2, I-00185 Roma, Italy lucio.pellacani@uniroma1.it. Fax: +39 06 490631. Phone: +39 0649913673

\section{Table of Contents}

General

Spectroscopic data for substrates $\mathbf{2}$ and $\mathbf{3} \quad$ S2

Spectroscopic data for aziridines $\mathbf{4 b - d} \quad$ S2

Spectroscopic data for aziridines $\mathbf{5 b}-\mathbf{d} \quad$ S3

Spectroscopic data for aziridines $\mathbf{6 b}-\mathbf{d} \quad \mathrm{S} 4$

Spectroscopic data for vinyl carbamates 7c-d and $\mathbf{8 d} \quad$ S4

Spectroscopic data for vinyl carbamates 11b-d $\quad$ S4

${ }^{1} \mathrm{H}$ NMR spectrum of compound $\mathbf{1} \quad$ S6

$\begin{array}{ll}{ }^{1} \mathrm{H} \text { NMR spectrum of compound } 2 & \text { S7 }\end{array}$

${ }^{13} \mathrm{C}$ NMR spectrum of compound $\mathbf{3} \quad$ S8

${ }^{1}$ H NMR spectrum of compound 4a $\quad$ S9

$\begin{array}{lr}{ }^{13} \mathrm{C} \text { NMR spectrum of compound } \mathbf{4 b} & \mathrm{S} 10\end{array}$

${ }^{1} \mathrm{H}$ NMR spectrum of compound $\mathbf{4 c} \quad \mathrm{S} 11$

$\begin{array}{ll}{ }^{13} \mathrm{C} \text { NMR spectrum of compound } \mathbf{4 d} & \mathrm{S} 12\end{array}$

${ }^{1} \mathrm{H}$ NMR spectrum of compound $\mathbf{5 a} \quad \mathrm{S} 13$

$\begin{array}{ll}{ }^{1} \mathrm{H} \text { NMR spectrum of compound } \mathbf{5 b} & \mathrm{S} 14\end{array}$

$\begin{array}{ll}{ }^{1} \mathrm{H} \text { NMR spectrum of compound } \mathbf{5 c} & \mathrm{S} 15\end{array}$

$\begin{array}{ll}{ }^{1} \mathrm{H} \text { NMR spectrum of compound } \mathbf{5 d} & \text { S16 }\end{array}$

$\begin{array}{ll}{ }^{1} \mathrm{H} \text { NMR spectrum of compound } \mathbf{6 a} & \text { S17 }\end{array}$

$\begin{array}{ll}{ }^{1} \mathrm{H} \text { NMR spectrum of compound } \mathbf{6} \mathbf{b} & \text { S18 }\end{array}$

$\begin{array}{ll}{ }^{1} \mathrm{H} \text { NMR spectrum of compound } \mathbf{6 c} & \text { S19 }\end{array}$

$\begin{array}{ll}{ }^{1} \text { H NMR spectrum of compound } \mathbf{6 d} & \text { S20 }\end{array}$

$\begin{array}{ll}{ }^{1} \mathrm{H} \text { NMR spectrum of compound 7a } & \text { S21 }\end{array}$

${ }^{13} \mathrm{C}$ NMR spectrum of compound 7a $\quad$ S22

${ }^{19}$ F NMR spectrum of compound 7c $\quad$ S23

${ }^{19}$ F NMR spectrum of compound 7d $\quad$ S24

${ }^{19}$ F NMR spectrum of compound 8a $\quad$ S25

${ }^{19}$ F NMR spectrum of compound 8d $\quad$ S26

$\begin{array}{ll}{ }^{13} \mathrm{C} \text { NMR spectrum of compound 11a } & \text { S27 }\end{array}$

${ }^{1} \mathrm{H}$ NMR spectrum of compound 11b $\quad$ S28

${ }^{13}$ C NMR spectrum of compound 11c $\quad$ S29

${ }^{1} \mathrm{H}$ NMR spectrum of compound 11d $\quad$ S30 
General. GC analyses were performed on a capillary column (methyl silicone, $12.5 \mathrm{~m} \times 0.2 \mathrm{~mm}$ ). GC MS were performed using a quadrupole mass spectrometer on a capillary column (phenyl methyl silicone, $30 \mathrm{~m} \times 0.25 \mathrm{~mm})$. IR spectra were recorded on a FTIR spectrophotometer in $\mathrm{CHCl}_{3}$ as the solvent. ${ }^{1} \mathrm{H}$ NMR and ${ }^{13} \mathrm{C}$ NMR spectra were recorded at 200 or 300 and 50 or $75 \mathrm{MHz}$, respectively. $\mathrm{CDCl}_{3}$ was used as the solvent and $\mathrm{CHCl}_{3}$ as the internal standard. ${ }^{19} \mathrm{~F}$ NMR spectra were recorded at 282.2 $\mathrm{MHz}$, using $\mathrm{CDCl}_{3}$ as the solvent and $\mathrm{C}_{6} \mathrm{~F}_{6}$ as the internal standard; chemical shifts are given in ppm relative to $\mathrm{CFCl}_{3}$. ESI MS analyses were performed using a quadrupole-time of flight (TOF) mass spectrometer equipped with an ESI source and a syringe pump. Melting points were determined with a Büchi apparatus and are uncorrected. Analytical thin-layer chromatography (TLC) was carried out on precoated $(0.25 \mathrm{~mm})$ silica gel plates using a mixture of EtOAc/hexane 20/80 as eluent.

The experiments were conducted in the positive ion mode. $\mathrm{NaH}$ (55-65\% suspension in mineral oil) was washed twice with pentane and dried under nitrogen. Anhydrous THF and toluene were used as such.

2: pale yellow liquid; IR: $1735,1662 \mathrm{~cm}^{-1} ;{ }^{1} \mathrm{H}$ NMR: $\delta 1.35(\mathrm{t}, J=7.2 \mathrm{~Hz}, 3 \mathrm{H}), 1.37(\mathrm{t}, J=7.2 \mathrm{~Hz}, 3$ $\mathrm{H}), 4.31(\mathrm{q}, J=7.2 \mathrm{~Hz}, 2 \mathrm{H}), 4.37(\mathrm{q}, J=7.2 \mathrm{~Hz}, 2 \mathrm{H}), 6.69(\mathrm{q}, J=1.2 \mathrm{~Hz}, 1 \mathrm{H}) ;{ }^{13} \mathrm{C} \mathrm{NMR:} \delta 13.1$, 14.6, 62.0, 62.6, $120.7(\mathrm{q}, J=273.3 \mathrm{~Hz}), 129.7(\mathrm{q}, J=5.4 \mathrm{~Hz}), 133.3(\mathrm{q}, J=32.9 \mathrm{~Hz}), 160.8,162.9$; ${ }^{19}$ F NMR: $\delta$-67.13; GC MS m/z $240\left(\mathrm{M}^{+}, 0.05\right), 195$ (18), 168 (13), $167(100), 150(10), 148(14), 147$ (16), 103 (12), 75 (14), 53 (11); HRMS-ESI (m/z) $[\mathrm{M}+\mathrm{Na}]^{+}$calcd for $\mathrm{C}_{9} \mathrm{H}_{11} \mathrm{~F}_{3} \mathrm{O}_{4}$ : 263.0507; found: 263.0514.

3: pale yellow liquid; IR: $2236(\mathrm{vw}), 1741,1644 \mathrm{~cm}^{-1} ;{ }^{1} \mathrm{H}$ NMR: $\delta 1.41(\mathrm{t}, J=7.2 \mathrm{~Hz}, 3 \mathrm{H}), 4.43(\mathrm{q}, J$ $=7.2 \mathrm{~Hz}, 2 \mathrm{H}), 6.49(\mathrm{q}, J=1.5 \mathrm{~Hz}, 1 \mathrm{H}) ;{ }^{13} \mathrm{C} \mathrm{NMR}: \delta 13.5,63.5,112.4,113.0(\mathrm{q}, J=6.6 \mathrm{~Hz}), 120.1$ $(\mathrm{q}, J=274.4 \mathrm{~Hz}), 139.3(\mathrm{q}, J=32.6 \mathrm{~Hz}), 158.4 ;{ }^{19} \mathrm{~F}$ NMR: $\delta$-67.10; GC MS $m / z 193\left(\mathrm{M}^{+}, 0.05\right), 166$ (11), 165 (13), 148 (100), 121 (11), 69 (29); HRMS-ESI (m/z) [M+Na] calcd for $\mathrm{C}_{7} \mathrm{H}_{6} \mathrm{~F}_{3} \mathrm{NO}_{2}$ : 216.0248; found: 216.0242 . 
4b: yellow oil, $R_{f}: 0.49$; IR: $1747 \mathrm{~cm}^{-1}$; ${ }^{1} \mathrm{H}$ NMR: $\delta 1.27(\mathrm{t}, J=7.2 \mathrm{~Hz}, 3 \mathrm{H}), 1.46(\mathrm{~s}, 9 \mathrm{H}), 2.32$ (s, 3 H), $3.47(\mathrm{~s}, 1 \mathrm{H}), 4.22-4.30(\mathrm{~m}, 2 \mathrm{H}) ;{ }^{13} \mathrm{C} \mathrm{NMR:} \delta 13.8,27.6,28.3,46.1,48.8(\mathrm{q}, J=34.9 \mathrm{~Hz}), 63.4$, 84.3, $121.3(\mathrm{q}, J=279.9 \mathrm{~Hz}), 154.7,161.2,198.8 ;{ }^{19} \mathrm{~F}$ NMR: $\delta$-68.85; GC MS $m / z 325\left(\mathrm{M}^{+}, 1\right), 152$ (100), 57 (92), 43 (10), 41 (15); HRMS-ESI $(m / z)[\mathrm{M}+\mathrm{Na}]^{+}$calcd for $\mathrm{C}_{13} \mathrm{H}_{18} \mathrm{~F}_{3} \mathrm{NO}_{5}$ : 348.1035; found: 348.1031.

4c: pale yellow oil, $R_{f}: 0.39$; IR: $1750 \mathrm{~cm}^{-1} ;{ }^{1} \mathrm{H}$ NMR: $\delta 1.27(\mathrm{t}, J=7.2 \mathrm{~Hz}, 3 \mathrm{H}), 2.34(\mathrm{~s}, 3 \mathrm{H}), 3.56$ (s, $1 \mathrm{H}), 4.20-4.29$ (m, $2 \mathrm{H}) ;$ 5.18-5.19 (m, $2 \mathrm{H}), 7.36$ (s, $5 \mathrm{H}) ;{ }^{13} \mathrm{C}$ NMR: $\delta 13.7,28.4,46.1,48.7$ (q, $J$ $=35.7 \mathrm{~Hz}), 63.5,69.6,121.0(\mathrm{q}, J=279.6 \mathrm{~Hz}), 128.4,128.5,128.6,134.1,156.1,160.8,198.0 ;{ }^{19} \mathrm{~F}$ NMR: $\delta$-69.31; GC MS m/z $359\left(\mathrm{M}^{+}, 1\right), 91(100)$; HRMS-ESI $(m / z)[\mathrm{M}+\mathrm{Na}]^{+}$calcd for $\mathrm{C}_{16} \mathrm{H}_{16} \mathrm{~F}_{3} \mathrm{NO}_{5}$ : 382.0878; found: 382.0869 .

4d: white low-melting solid; IR: $1751 \mathrm{~cm}^{-1} ;{ }^{1} \mathrm{H}$ NMR: $\delta 1.35(\mathrm{t}, J=7.2 \mathrm{~Hz}, 3 \mathrm{H}), 2.33(\mathrm{~s}, 3 \mathrm{H}), 3.52$ (s, $1 \mathrm{H}), 4.23-4.30(\mathrm{~m}, 1 \mathrm{H}), 4.34(\mathrm{q}, J=7.2 \mathrm{~Hz}, 2 \mathrm{H}), 4.51-4.55(\mathrm{~m}, 2 \mathrm{H}), 7.30-7.44(\mathrm{~m}, 4 \mathrm{H}), 7.58-$ 7.65 (m, $2 \mathrm{H}), 7.75-7.78(\mathrm{~m}, 2 \mathrm{H}) ;{ }^{13} \mathrm{C} \mathrm{NMR}: \delta 13.8,28.4,45.9,46.6,48.9(\mathrm{q}, J=35.8 \mathrm{~Hz}), 63.6,69.6$, $119.9,121.0(\mathrm{q}, J=279.9 \mathrm{~Hz}), 124.8,124.9,127.1,127.8,141.1,142,7,143.0,156.0,160.8,197.9$; ${ }^{19}$ F NMR: $\delta$-69.40; HRMS-ESI $(m / z)[\mathrm{M}+\mathrm{Na}]^{+}$calcd for $\mathrm{C}_{23} \mathrm{H}_{20} \mathrm{~F}_{3} \mathrm{NO}_{5}: 470.1191$; found: 470.1179 .

5b: pale yellow oil, $R_{f}: 0.45$; IR: $1749 \mathrm{~cm}^{-1} ;{ }^{1} \mathrm{H} \mathrm{NMR}: \delta 1.27(\mathrm{t}, J=7.2 \mathrm{~Hz}, 3 \mathrm{H}), 1.29(\mathrm{t}, J=7.2 \mathrm{~Hz}$, $3 \mathrm{H}), 1.45(\mathrm{~s}, 9 \mathrm{H}), 3.53(\mathrm{~s}, 1 \mathrm{H}), 4.22(\mathrm{q}, J=7.2 \mathrm{~Hz}, 2 \mathrm{H}), 4.29(\mathrm{q}, J=7.2 \mathrm{~Hz}, 2 \mathrm{H}) ;{ }^{13} \mathrm{C} \mathrm{NMR}: \delta 13.9$, $14.0,27.6,40.9,48.6(\mathrm{q}, J=35.5 \mathrm{~Hz}), 62.5,63.2,84.2,121.3(\mathrm{q}, J=280.5 \mathrm{~Hz}), 154.7,160.7,163.8$; ${ }^{19}$ F NMR: $\delta$-69.06; GC MS m/z $340\left(\mathrm{M}^{+}-15,<1\right), 296$ (11), 182 (14), 154 (10), 57 (100), 41 (11); HRMS-ESI $(m / z)[\mathrm{M}+\mathrm{Na}]^{+}$calcd for $\mathrm{C}_{14} \mathrm{H}_{20} \mathrm{~F}_{3} \mathrm{NO}_{6}$ : 378.1140; found: 378.1133 .

5c: yellow oil, $R_{f}: 0.39$; IR: $1748 \mathrm{~cm}^{-1} ;{ }^{1} \mathrm{H}$ NMR: $\delta 1.31(\mathrm{t}, J=7.2 \mathrm{~Hz}, 3 \mathrm{H}), 1.32(\mathrm{t}, J=7.2 \mathrm{~Hz}, 3 \mathrm{H})$, $3.63(\mathrm{~s}, 1 \mathrm{H}), 4.27(\mathrm{q}, J=7.2 \mathrm{~Hz}, 2 \mathrm{H}), 4.32(\mathrm{q}, J=7.2 \mathrm{~Hz}, 2 \mathrm{H}), 5.21-5.22(\mathrm{~m}, 2 \mathrm{H}), 7.37(\mathrm{~s}, 5 \mathrm{H}) ;{ }^{13} \mathrm{C}$ NMR: $\delta 13.7,13.8,40.9,48.5(\mathrm{q}, J=36.0 \mathrm{~Hz}), 62.6,63.3,69.5,121.1(\mathrm{q}, J=280.3 \mathrm{~Hz}), 128.3,128.5$, 
134.2, 155.8, 160.3, 163.3; ${ }^{19} \mathrm{~F}$ NMR: $\delta$-69.66; GC MS m/z $389\left(\mathrm{M}^{+}, 0.01\right), 210(60), 182(30), 107$ (46), 91 (100); HRMS-ESI (m/z) [M+Na] ${ }^{+}$calcd for $\mathrm{C}_{17} \mathrm{H}_{18} \mathrm{~F}_{3} \mathrm{NO}_{6}$ : 412.0984; found: 412.0973.

5d: white solid, mp: $66-68{ }^{\circ} \mathrm{C}$ (pentane/ $\left.\mathrm{CH}_{2} \mathrm{Cl}_{2}\right)$; IR: $1748 \mathrm{~cm}^{-1} ;{ }^{1} \mathrm{H}$ NMR: $\delta 1.35(\mathrm{t}, J=7.2 \mathrm{~Hz}, 3 \mathrm{H})$, $1.39(\mathrm{t}, J=7.2 \mathrm{~Hz}, 3 \mathrm{H}), 3.66(\mathrm{~s}, 1 \mathrm{H}), 4.24-4.33(\mathrm{~m}, 3 \mathrm{H}), 4.35-4.43(\mathrm{~m}, 2 \mathrm{H}), 4.45-4.51(\mathrm{~m}, 2 \mathrm{H})$, 7.32-7.45 (m, $4 \mathrm{H}), 7.62-7.69$ (m, $2 \mathrm{H}), 7.77-7.80(\mathrm{~m}, 2 \mathrm{H}) ;{ }^{13} \mathrm{C}$ NMR: $\delta 13.9,14.0,40.8,46.5,48.7$ (q, $J=35.8 \mathrm{~Hz}), 62.8,63.4,69.7,119.9,121.1(\mathrm{q}, J=280.5 \mathrm{~Hz}), 125.0,125.1,127.0,127.8,141.1,142.8$, 143.0, 155.7, 160.3, 163.4; ${ }^{19} \mathrm{~F}$ NMR: $\delta$-69.81; HRMS-ESI $(m / z)[\mathrm{M}+\mathrm{Na}]^{+}$calcd for $\mathrm{C}_{24} \mathrm{H}_{22} \mathrm{~F}_{3} \mathrm{NO}_{6}$ : 500.1297; found: 500.1287.

6b: pale yellow oil, $R_{f}: 0.55$; IR: $2253(\mathrm{vw}), 1758 \mathrm{~cm}^{-1} ;{ }^{1} \mathrm{H}$ NMR: $\delta 1.38(\mathrm{t}, J=7.2 \mathrm{~Hz}, 3 \mathrm{H}), 1.51$ (s, $9 \mathrm{H}), 3.49$ (s, $1 \mathrm{H}), 4.33-4.50(\mathrm{~m}, 2 \mathrm{H}) ;{ }^{13} \mathrm{C} \mathrm{NMR}: \delta 13.9,27.7,29.5,47.0(\mathrm{q}, J=36.6 \mathrm{~Hz}), 64.7,85.6$, 110.6, $120.2(\mathrm{q}, J=278.3 \mathrm{~Hz}), 152.0,159.5 ;{ }^{19} \mathrm{~F} \mathrm{NMR:} \delta$-72.06; GC MS $m / z, 308\left(\mathrm{M}^{+}, 0.01\right), 207(20)$, 57 (100), 41 (16); HRMS-ESI $(\mathrm{m} / z)[\mathrm{M}+\mathrm{Na}]^{+}$calcd for $\mathrm{C}_{12} \mathrm{H}_{15} \mathrm{~F}_{3} \mathrm{~N}_{2} \mathrm{O}_{4}: 331.0882$; found: 331.0890 .

6c: pale yellow oil, $R_{f}: 0.44$; IR: $2254(\mathrm{vw}), 1760 \mathrm{~cm}^{-1} ;{ }^{1} \mathrm{H}$ NMR: $\delta 1.29$ (t, $\left.J=7.2 \mathrm{~Hz}, 3 \mathrm{H}\right), 3.49$ (s, 1 $\mathrm{H}), 4.21-4.41(\mathrm{~m}, 2 \mathrm{H}), 5.26(\mathrm{~s}, 2 \mathrm{H}), 7.37$ (s, $5 \mathrm{H}) ;{ }^{13} \mathrm{C} \mathrm{NMR}: \delta 13.8,29.3,47.6(\mathrm{q}, J=36.7 \mathrm{~Hz}), 64.7$, $70.2,110.2,120.0(\mathrm{q}, J=278.3 \mathrm{~Hz}), 128.5,128.6,128.8,133.8,153.7,159.2 ;{ }^{19} \mathrm{~F} \mathrm{NMR}: \delta-72.33 ; \mathrm{GC}$ MS m/z $342\left(\mathrm{M}^{+}, 0.07\right), 225$ (22), 107 (72), 91 (100), 90 (10), 79 (19), 65 (12); HRMS-ESI (m/z) $[\mathrm{M}+\mathrm{Na}]^{+}$calcd for $\mathrm{C}_{15} \mathrm{H}_{13} \mathrm{~F}_{3} \mathrm{~N}_{2} \mathrm{O}_{4}: 365.0725$; found: 365.0717 .

6d: white solid, mp: $174-176{ }^{\circ} \mathrm{C}$ (pentane/ $\left.\mathrm{CH}_{2} \mathrm{Cl}_{2}\right)$; IR: $2254(\mathrm{vw}), 1761 \mathrm{~cm}^{-1} ;{ }^{1} \mathrm{H}$ NMR: $\delta 1.32(\mathrm{t}, J=$ 7.2 Hz, 3 H), 3.60 (s, 1 H), 4.27-4.41 (m, 3 H), 4.61-4.67 (m, 2 H), 7.31-7.37 (m, 2 H), 7.41-7.46 (m, 2 $\mathrm{H}), 7.56-7.61(\mathrm{~m}, 2 \mathrm{H}), 7.72-7.78(\mathrm{~m}, 2 \mathrm{H}) ;{ }^{13} \mathrm{C} \mathrm{NMR}: \delta 13.8,29.2,46.4,47.1(\mathrm{q}, J=37.8 \mathrm{~Hz}), 64.9$, $70.3,110.1,120.0(\mathrm{q}, J=278.3 \mathrm{~Hz}), 120.1,124.8,125.0,127.1,127.9,141.2,142.5,142.9,153.7$, 
159.3; ${ }^{19} \mathrm{~F}$ NMR: $\delta$-72.46; HRMS-ESI $(\mathrm{m} / \mathrm{z})[\mathrm{M}+\mathrm{Na}]^{+}$calcd for $\mathrm{C}_{22} \mathrm{H}_{17} \mathrm{~F}_{3} \mathrm{~N}_{2} \mathrm{O}_{4}$ : 453.1038; found: 453.1036.

7c: ${ }^{19}$ F NMR: $\delta$-55.84; GC MS m/z $359\left(\mathrm{M}^{+}, 0.5\right), 91(100)$.

7d: ${ }^{19}$ F NMR: $\delta-55.88$.

8d: ${ }^{19}$ F NMR: $\delta-58.23$.

11b: colorless oil, $R_{f}: 0.52$; IR: $1752 \mathrm{~cm}^{-1}$; ${ }^{1} \mathrm{H}$ NMR: $\delta 1.28-1.38(\mathrm{~m}, 6 \mathrm{H}), 1.46(\mathrm{~s}, 9 \mathrm{H}), 3.70(\mathrm{q}, J=$ $5.1 \mathrm{~Hz}, 1 \mathrm{H}), 4.27-4.36(\mathrm{~m}, 4 \mathrm{H}) ;{ }^{13} \mathrm{C} \mathrm{NMR}: \delta 13.9,14.0,27.8,44.6(\mathrm{q}, J=41.5 \mathrm{~Hz}), 48.5,62.6,63.9$, 84.1, $121.2(\mathrm{q}, J=274.9 \mathrm{~Hz}), 155.1,162.0,163.2 ;{ }^{19} \mathrm{~F}$ NMR: $\delta$-71.92 (d, $\left.J=4.8 \mathrm{~Hz}\right)$; GC MS $m / z 340$ $\left(\mathrm{M}^{+}-15,<1\right), 296(7), 182(8), 57(100), 41(12)$; HRMS-ESI $(m / z)[\mathrm{M}+\mathrm{Na}]^{+}$calcd for $\mathrm{C}_{14} \mathrm{H}_{20} \mathrm{~F}_{3} \mathrm{NO}_{6}$ : 378.1140; found: 378.1144 .

11c: colorless oil, $R_{f}: 0.44$; IR: $1754 \mathrm{~cm}^{-1} ;{ }^{1} \mathrm{H}$ NMR $\delta 1.19(\mathrm{t}, J=7.2 \mathrm{~Hz}, 3 \mathrm{H}), 1.30(\mathrm{t}, J=7.2 \mathrm{~Hz}, 3$ H), $3.77(\mathrm{q}, J=4.8 \mathrm{~Hz}, 1 \mathrm{H}), 4.11(\mathrm{q}, J=7.2 \mathrm{~Hz}, 2 \mathrm{H}), 4.32(\mathrm{q}, J=7.2 \mathrm{~Hz}, 2 \mathrm{H}), 5.13-5.25(\mathrm{~m}, 2 \mathrm{H})$, $7.35(\mathrm{~s}, 5 \mathrm{H}) ;{ }^{13} \mathrm{C}$ NMR: $\delta$ 13.6, $44.4(\mathrm{q}, J=41.8 \mathrm{~Hz}), 48.3,62.7,64.0,69.4,121.1(\mathrm{q}, J=275.3 \mathrm{~Hz})$, $128.4,128.5,128.6,134.5,156.7,161.8,163.0 ;{ }^{19} \mathrm{~F} \mathrm{NMR}: \delta-71.47 ;$ GC MS $m / z 298\left(\mathrm{M}^{+}-91,<1\right), 214$ (51), 168 (32), 107 (25), 91 (100); HRMS-ESI (m/z) $[\mathrm{M}+\mathrm{Na}]^{+}$calcd for $\mathrm{C}_{17} \mathrm{H}_{18} \mathrm{~F}_{3} \mathrm{NO}_{6}$ : 412.0984; found: 412.0977 .

11d: yellow viscous oil, $R_{f}: 0.41$; IR: $1753 \mathrm{~cm}^{-1} ;{ }^{1} \mathrm{H} \mathrm{NMR}: \delta 1.27(\mathrm{t}, J=7.2 \mathrm{~Hz}, 3 \mathrm{H}), 1.39(\mathrm{t}, J=7.2$ Hz, $3 \mathrm{H}), 3.82$ (q, $J=4.8 \mathrm{~Hz}, 1 \mathrm{H}), 4.20-4.34(\mathrm{~m}, 3 \mathrm{H}), 4.38-4.46$ (m, $2 \mathrm{H}), 4.50-4.55$ (m, 2 H), 7.33$7.36(\mathrm{~m}, 2 \mathrm{H}), 7.38-7.45(\mathrm{~m}, 2 \mathrm{H}), 7.62-7.71(\mathrm{~m}, 2 \mathrm{H}), 7.73-7.79(\mathrm{~m}, 2 \mathrm{H}) ;{ }^{13} \mathrm{C}$ NMR: $\delta 13.8,13.9,44.6$ $(\mathrm{q}, J=41.2 \mathrm{~Hz}), 46.5,48.3,62.8,64.2,69.7,119.9,121.1(\mathrm{q}, J=274.8 \mathrm{~Hz}), 125.0,125.1,127.0$, $127.1,127.8,127.9,141.1,142.8,143.0,156.5,161.3,163.1 ;{ }^{19} \mathrm{~F} \mathrm{NMR:} \delta-71.43(\mathrm{~d}, J=6.2 \mathrm{~Hz})$; HRMS-ESI $(m / z)[\mathrm{M}+\mathrm{Na}]^{+}$calcd for $\mathrm{C}_{24} \mathrm{H}_{22} \mathrm{~F}_{3} \mathrm{NO}_{6}$ : 500.1297; found: 500.1304 . 


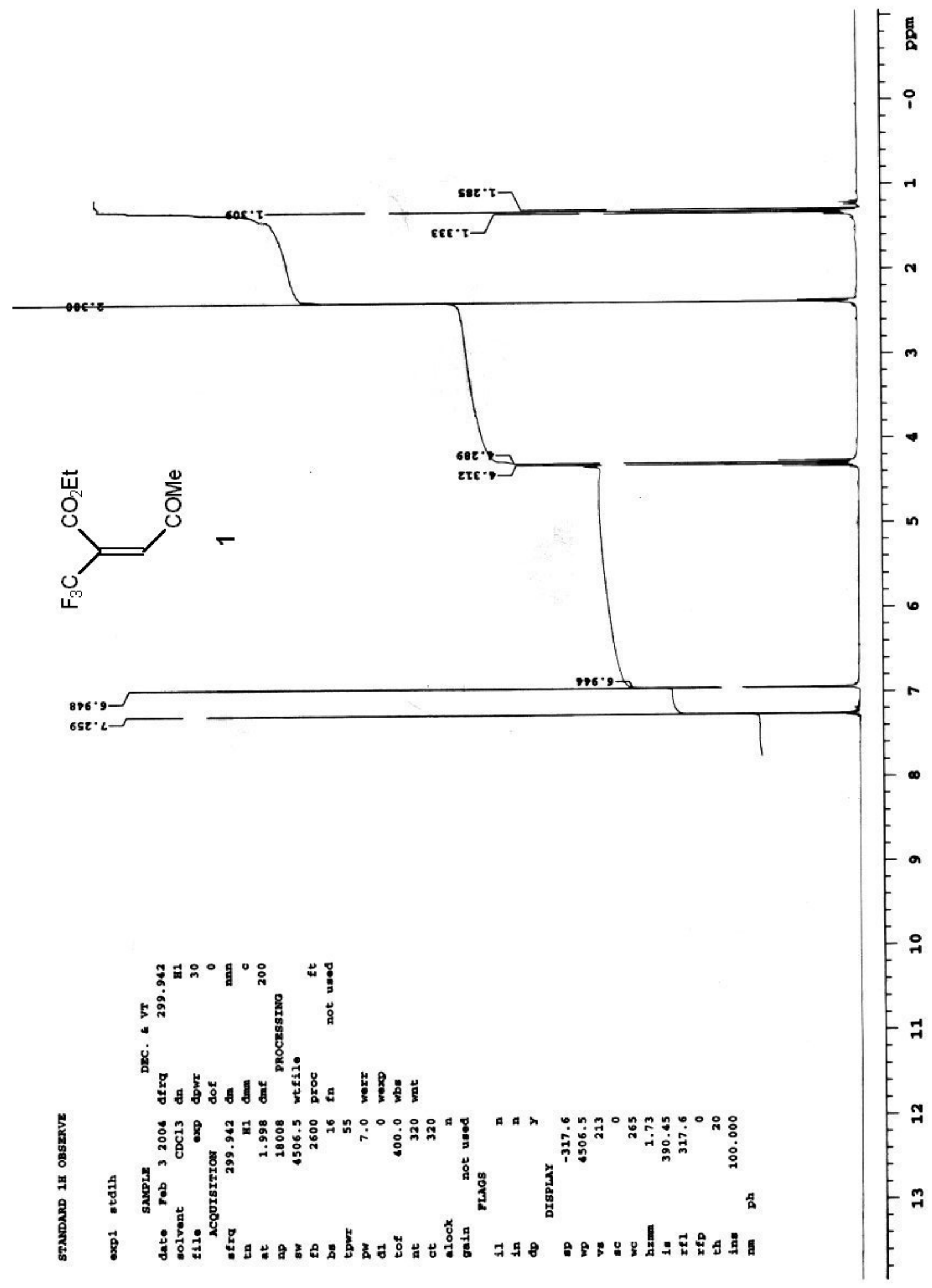




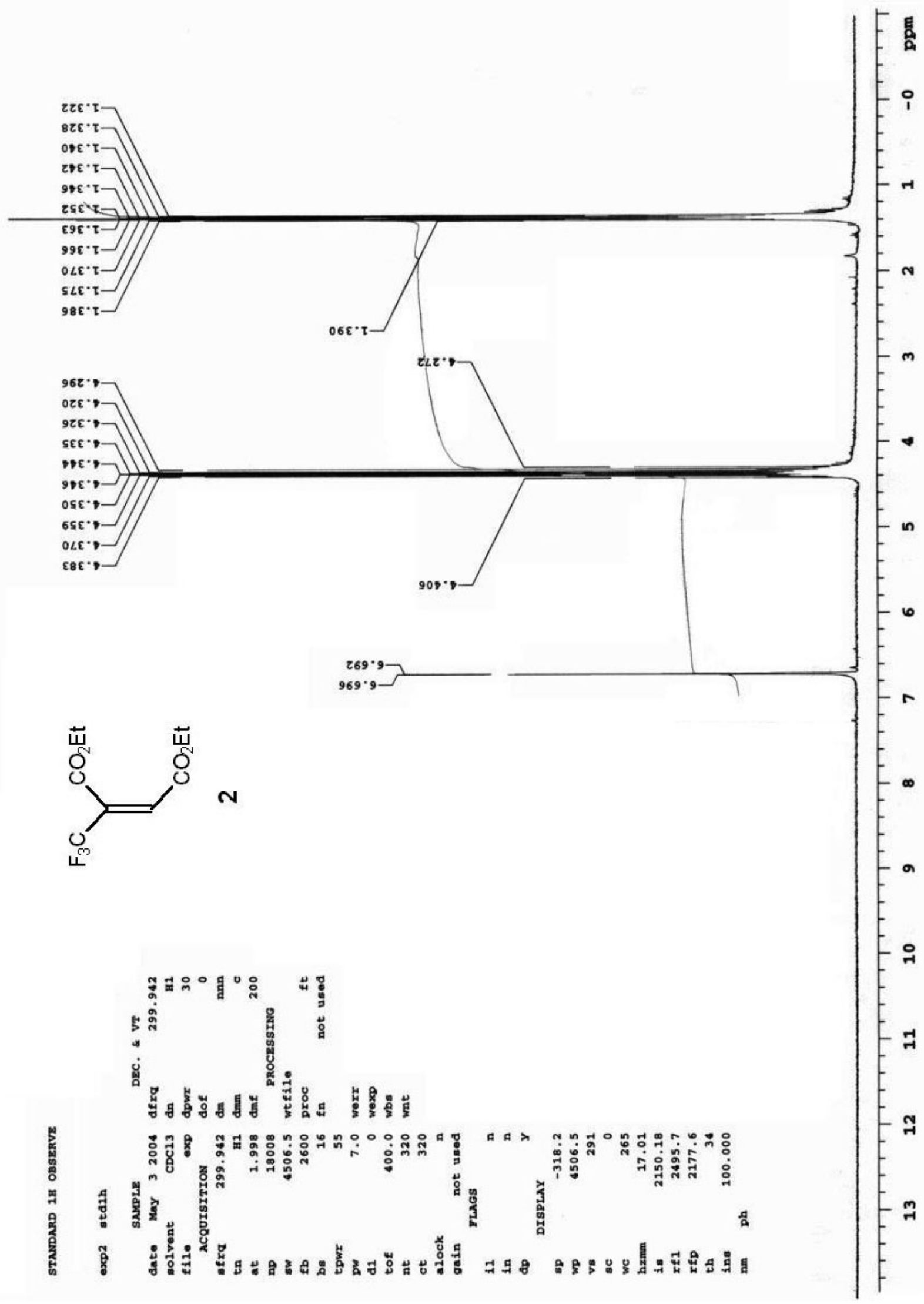




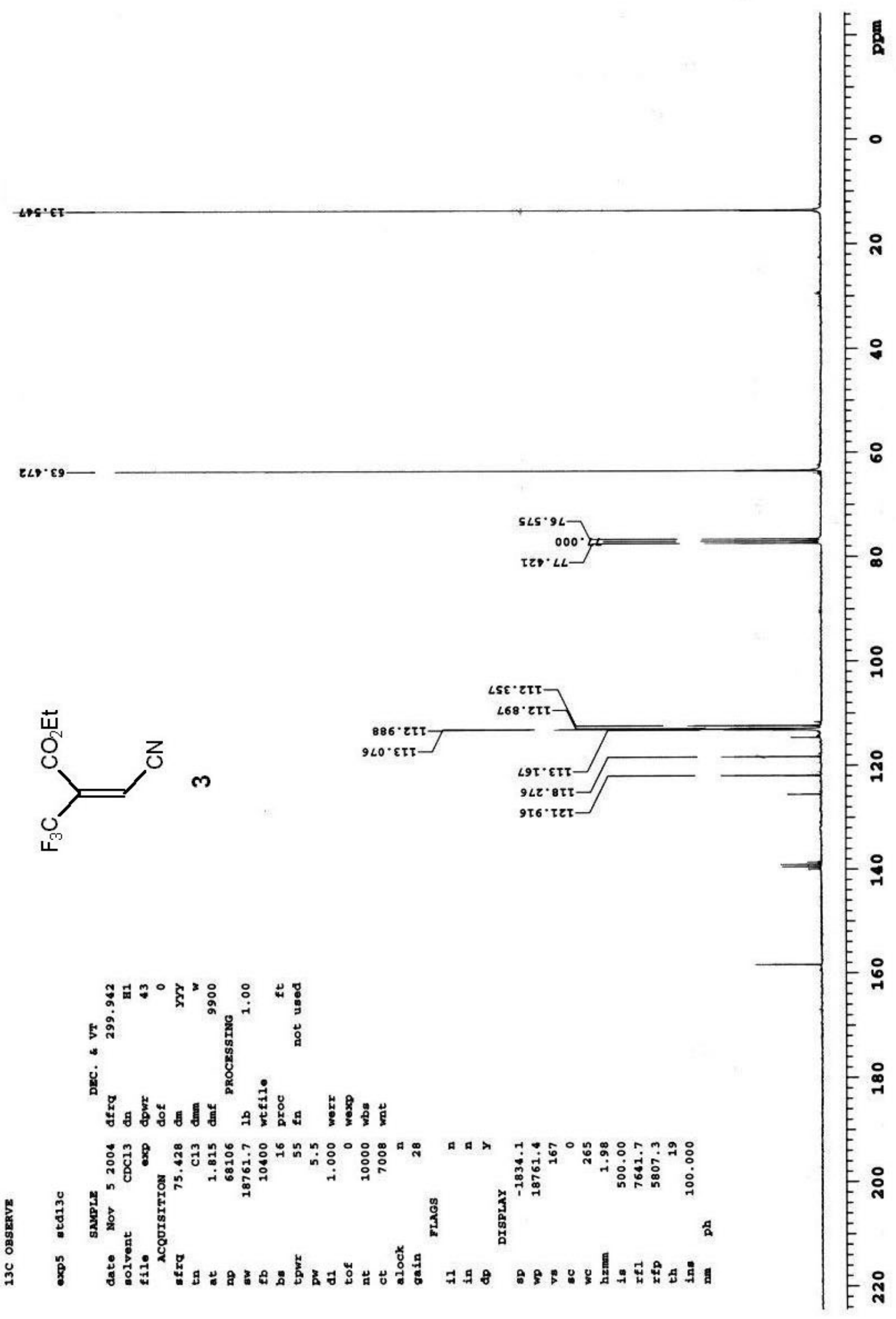

1 


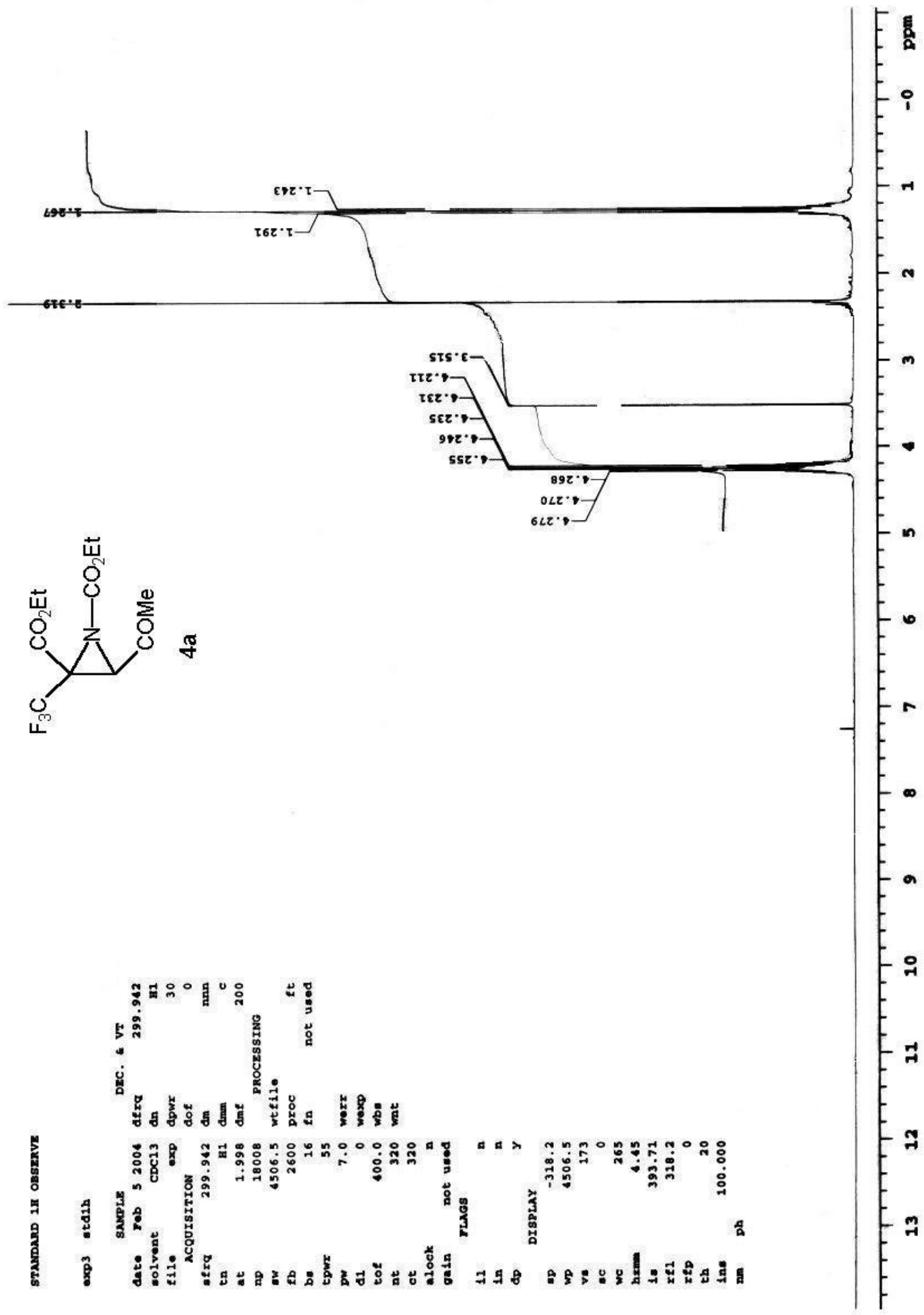


$56 L^{*} \mathrm{ET}$
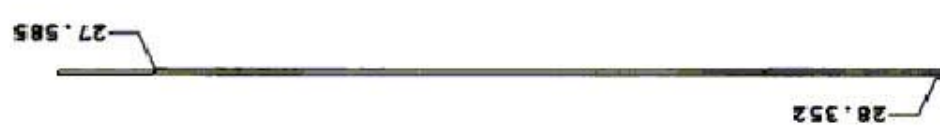

$640^{\circ} 94$

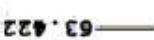
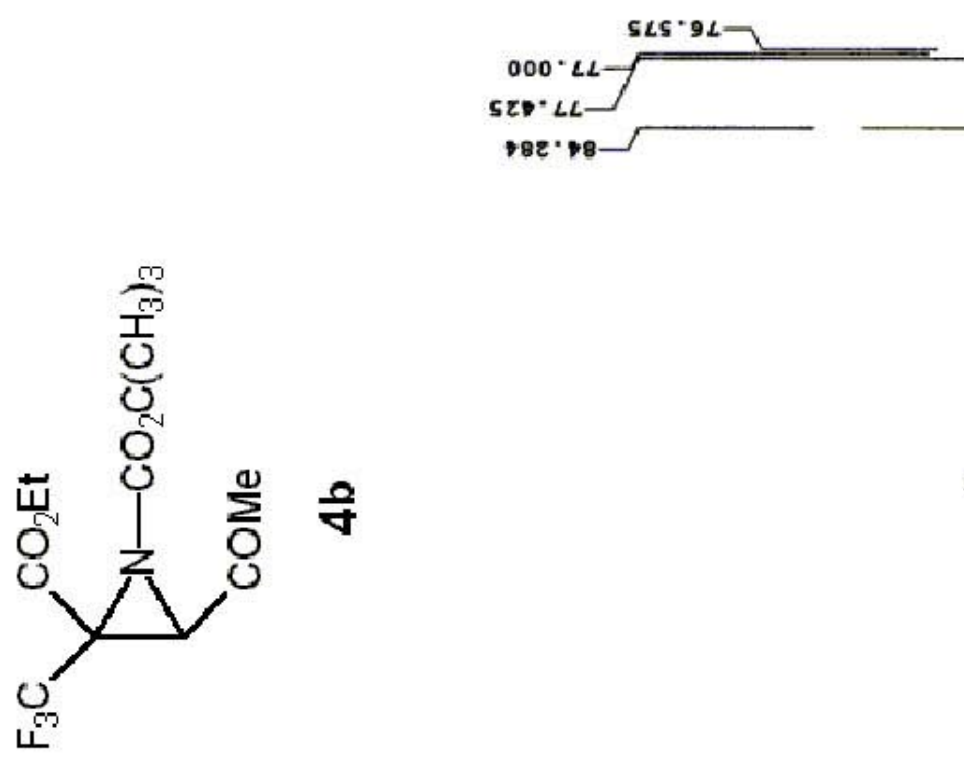

Z\$F* 6TT

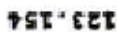

$2+4.55$

Сाน"T

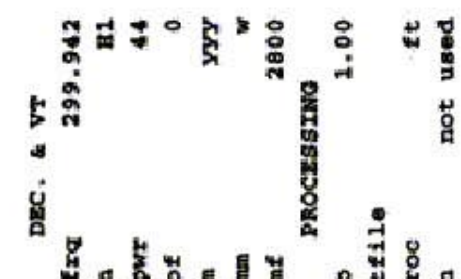

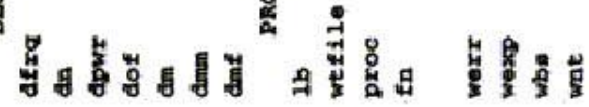

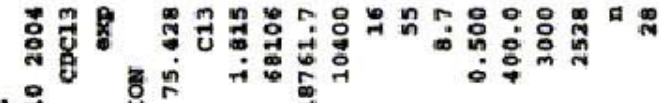

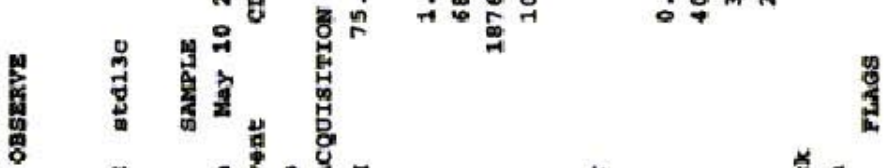

त व

$n$
0
0
0

界

면

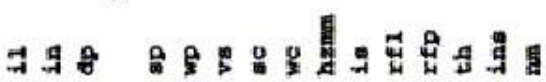




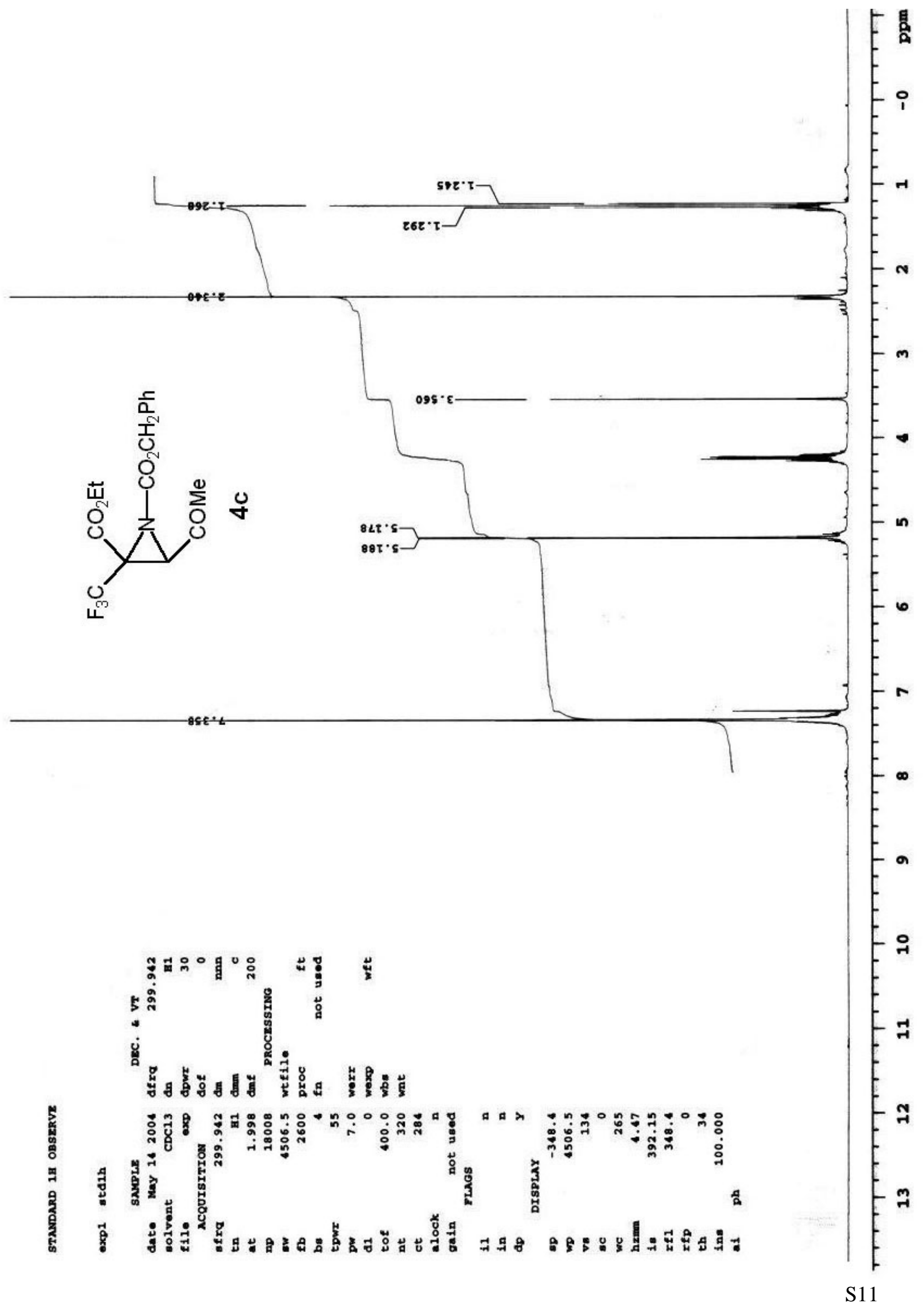




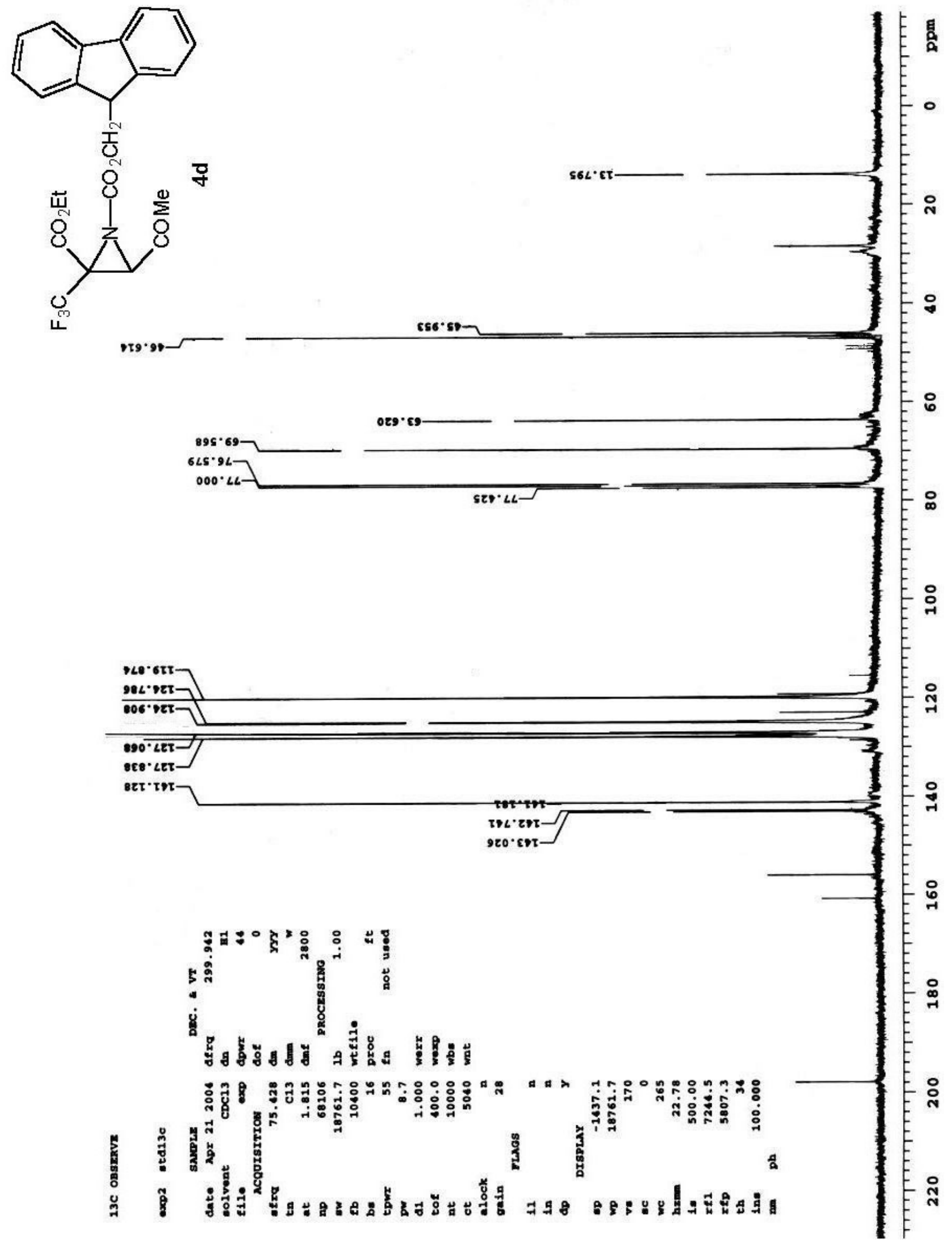




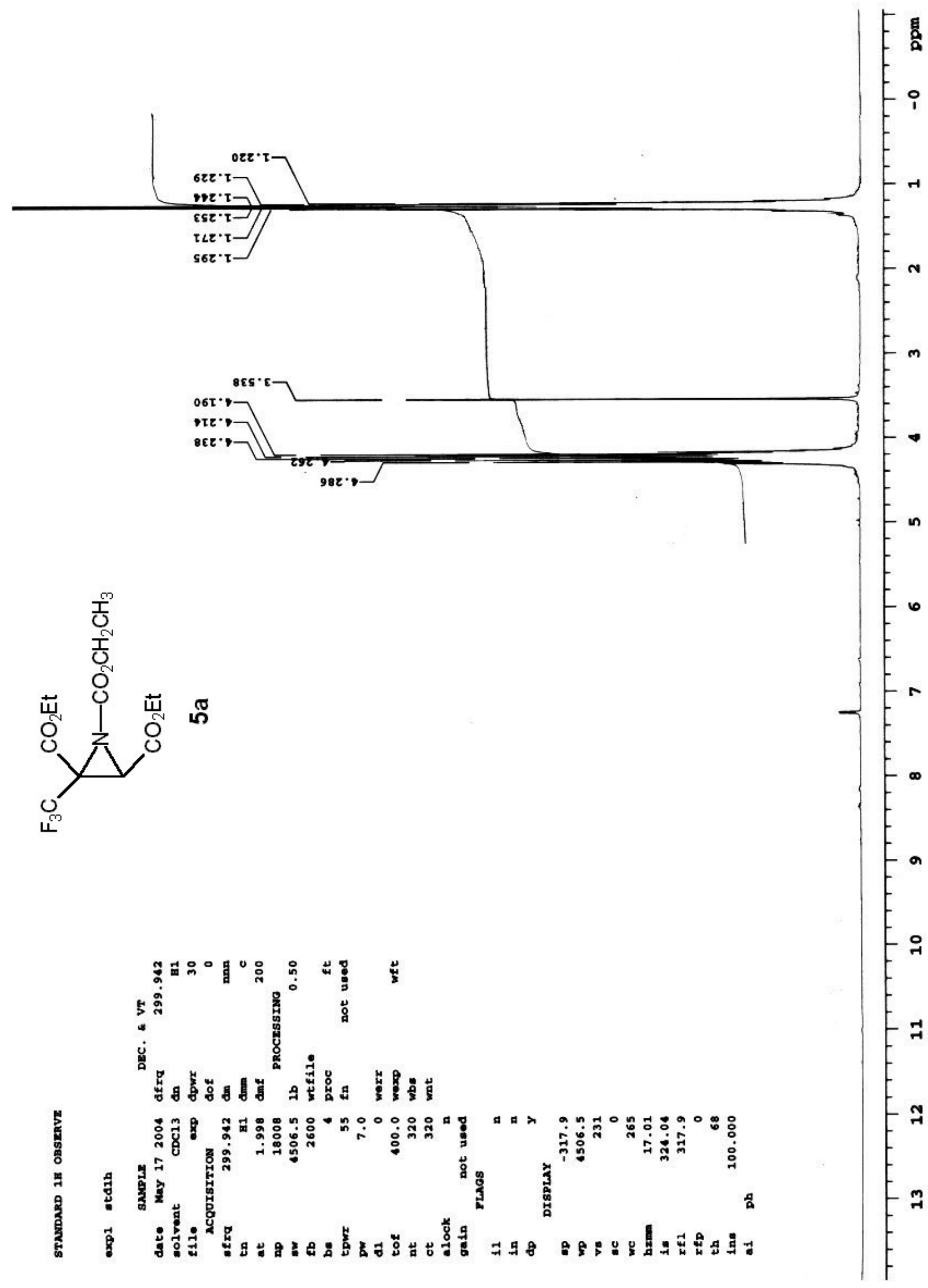




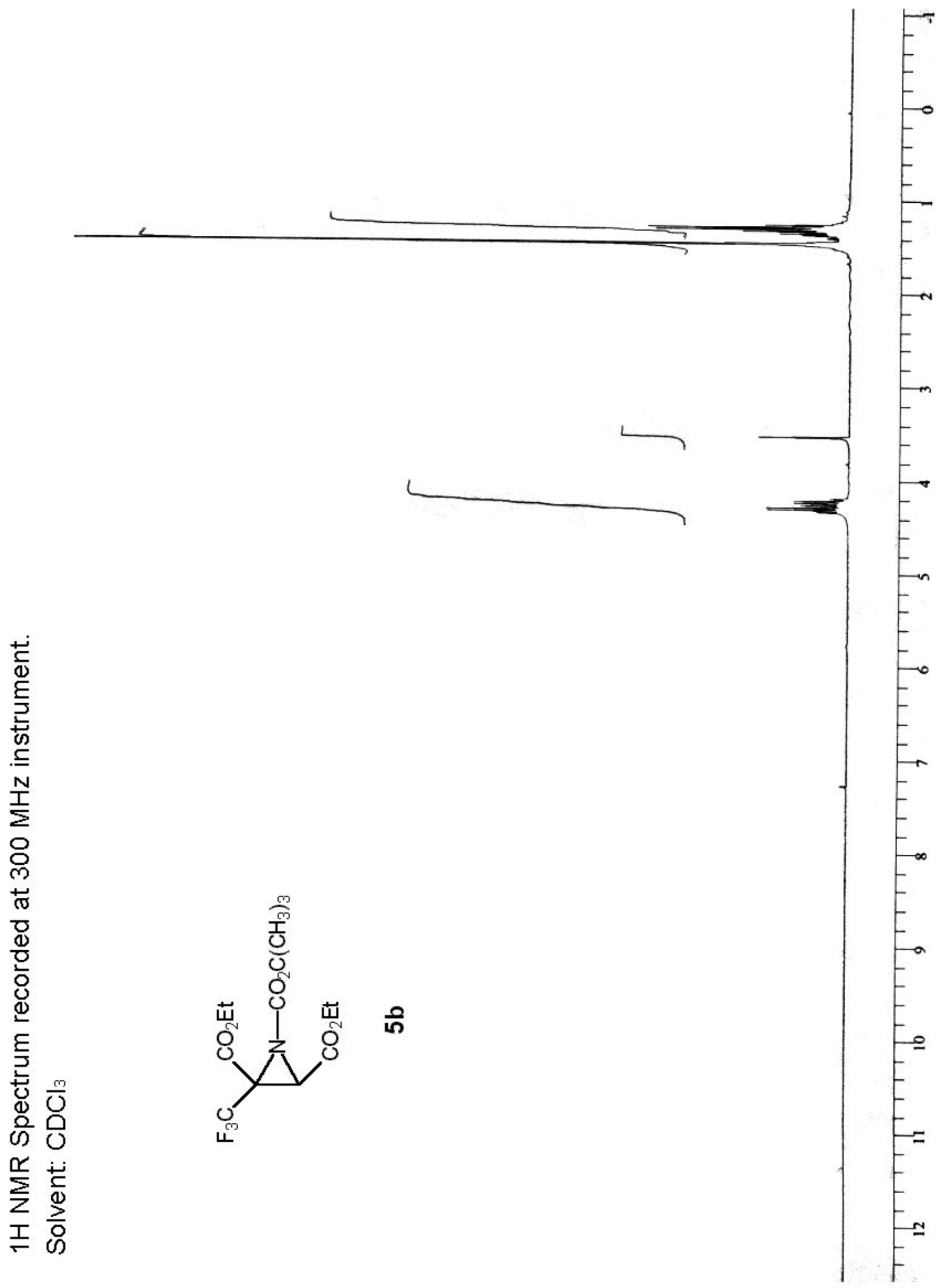




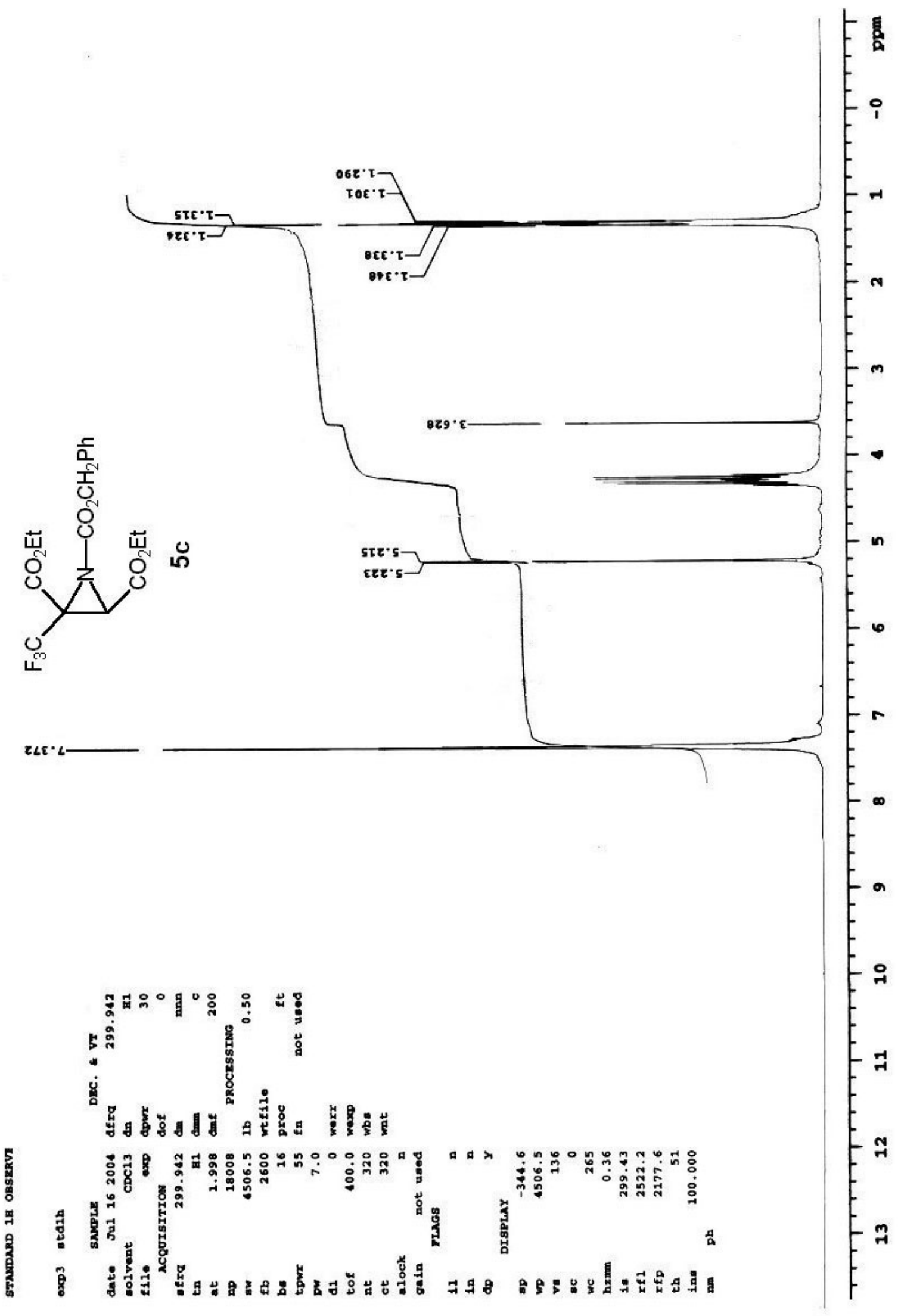




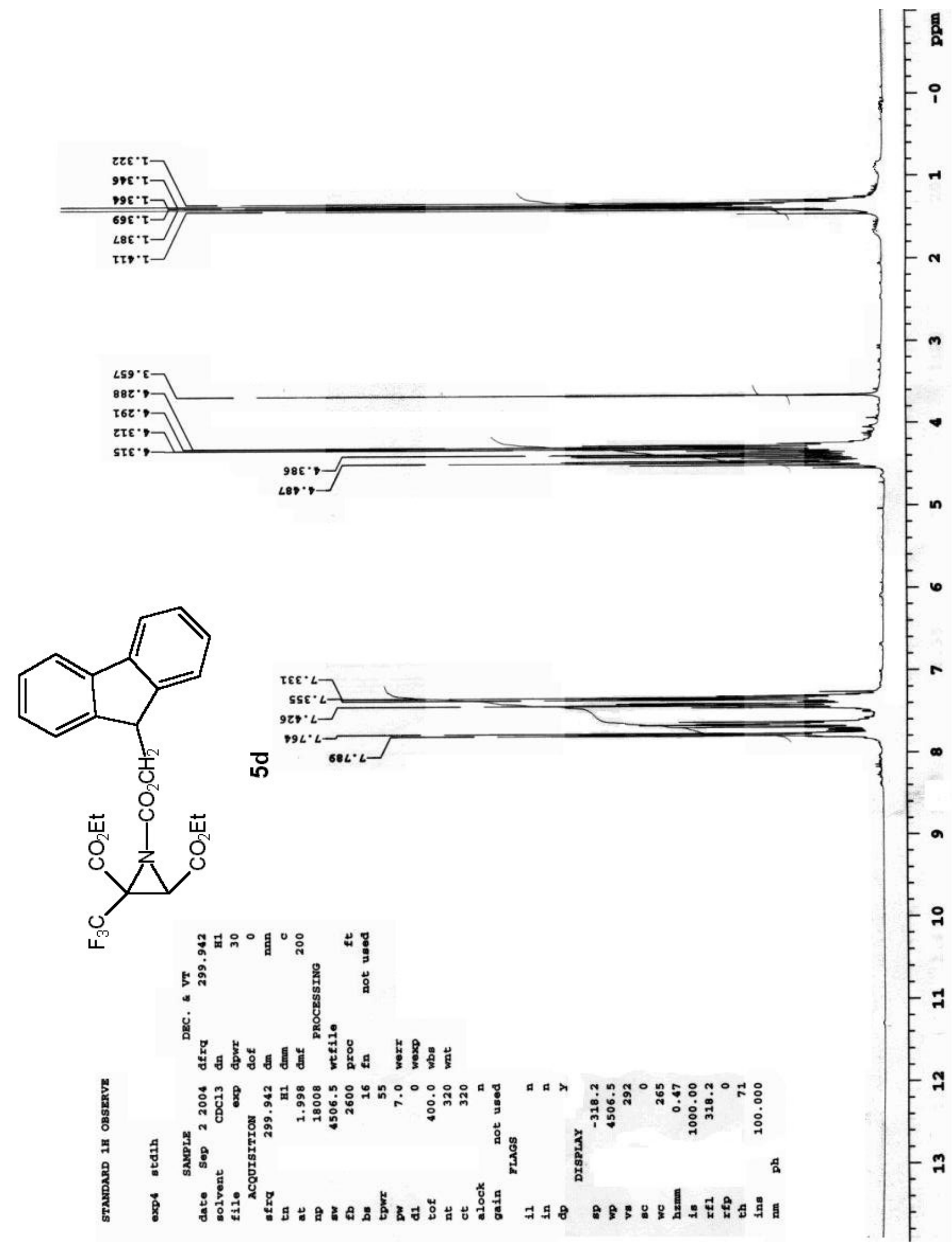




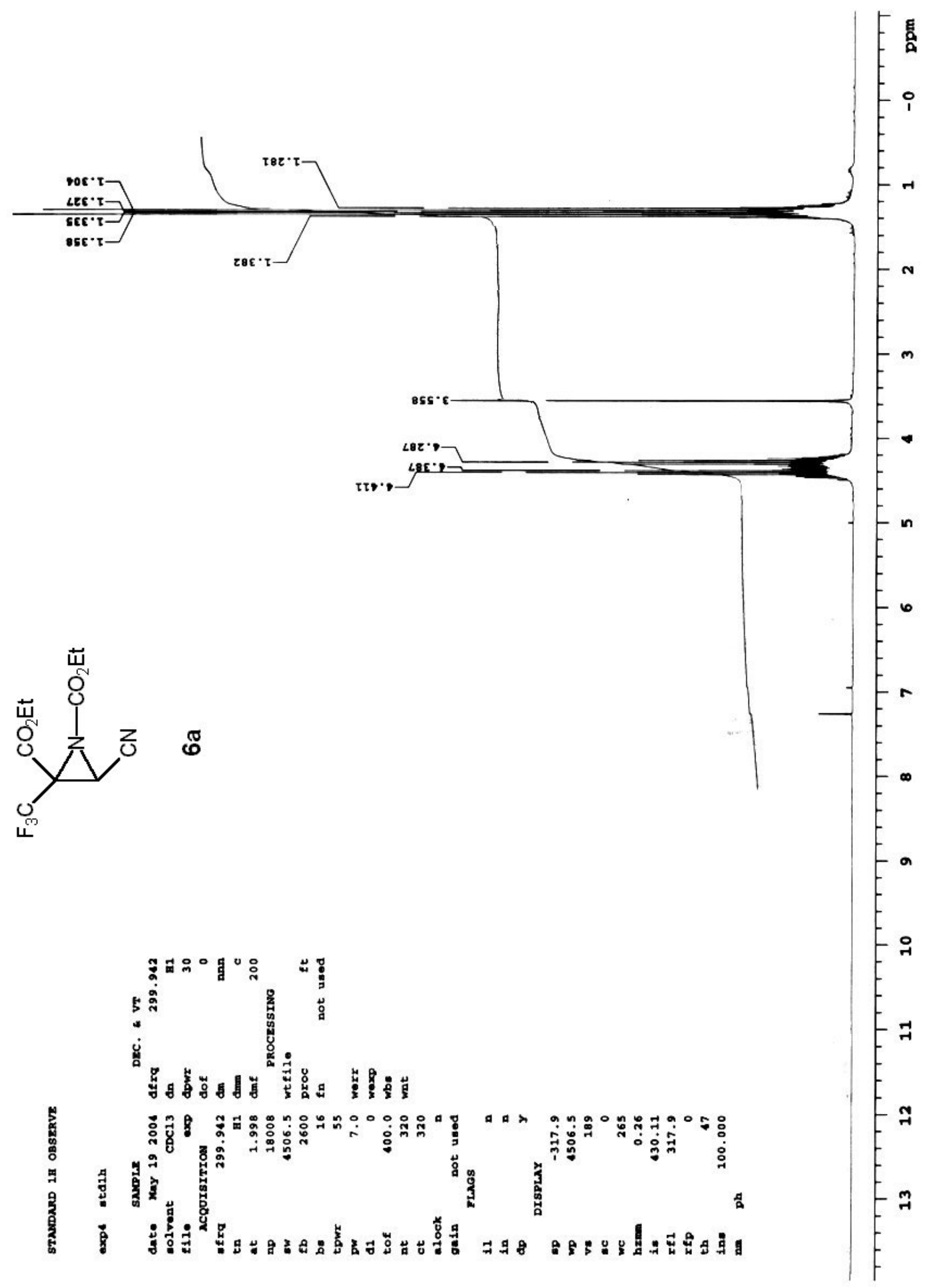




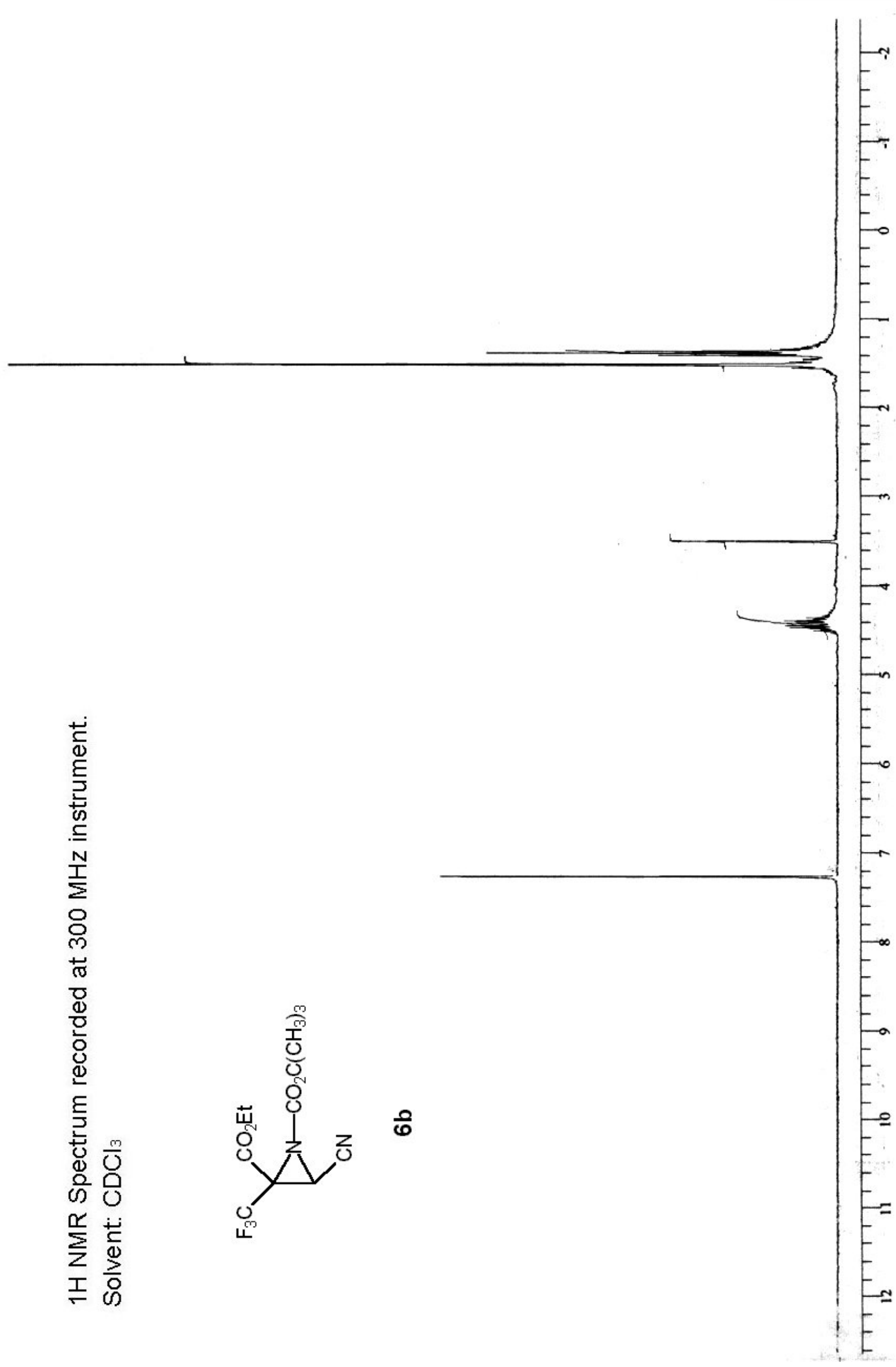




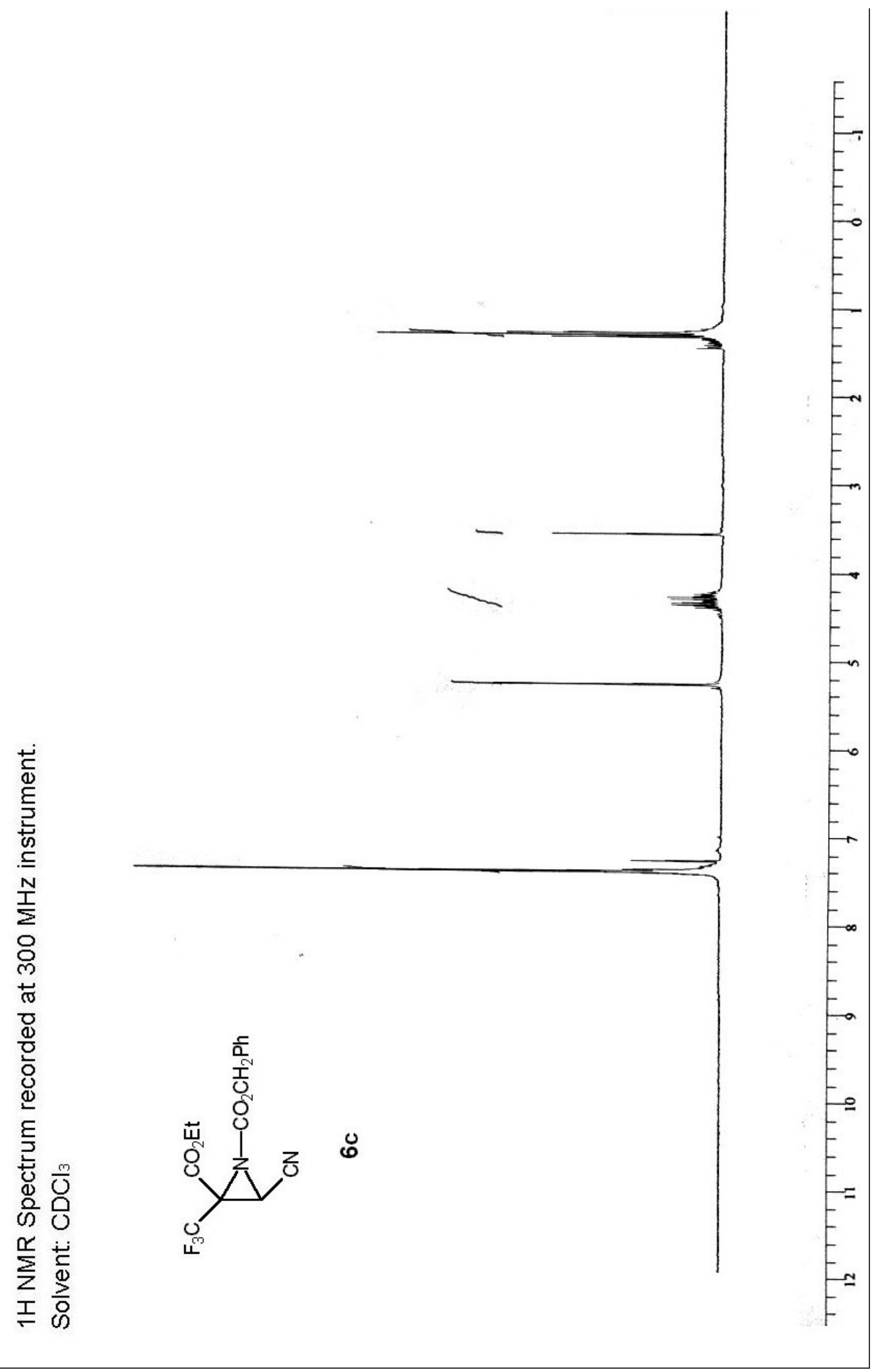




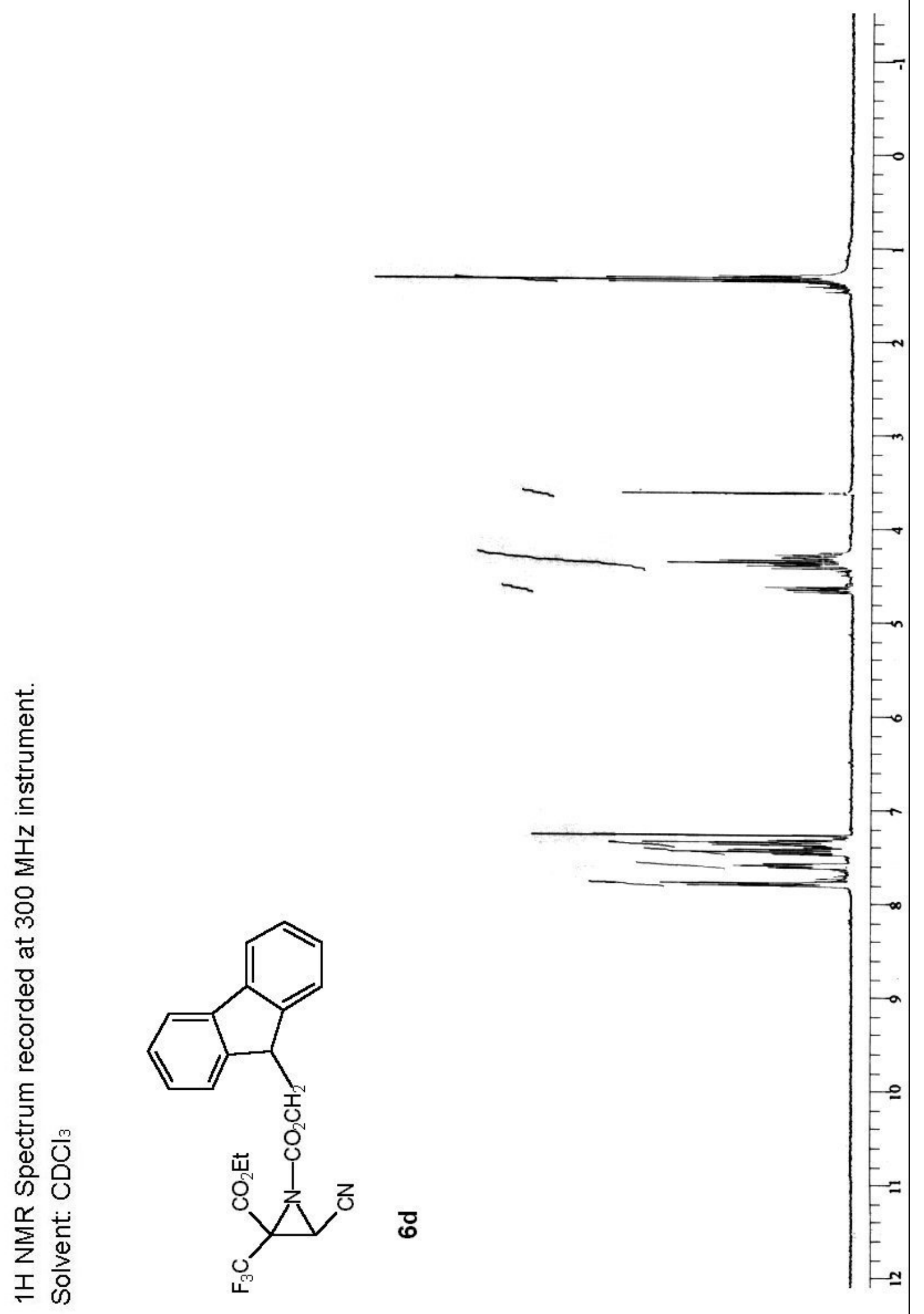




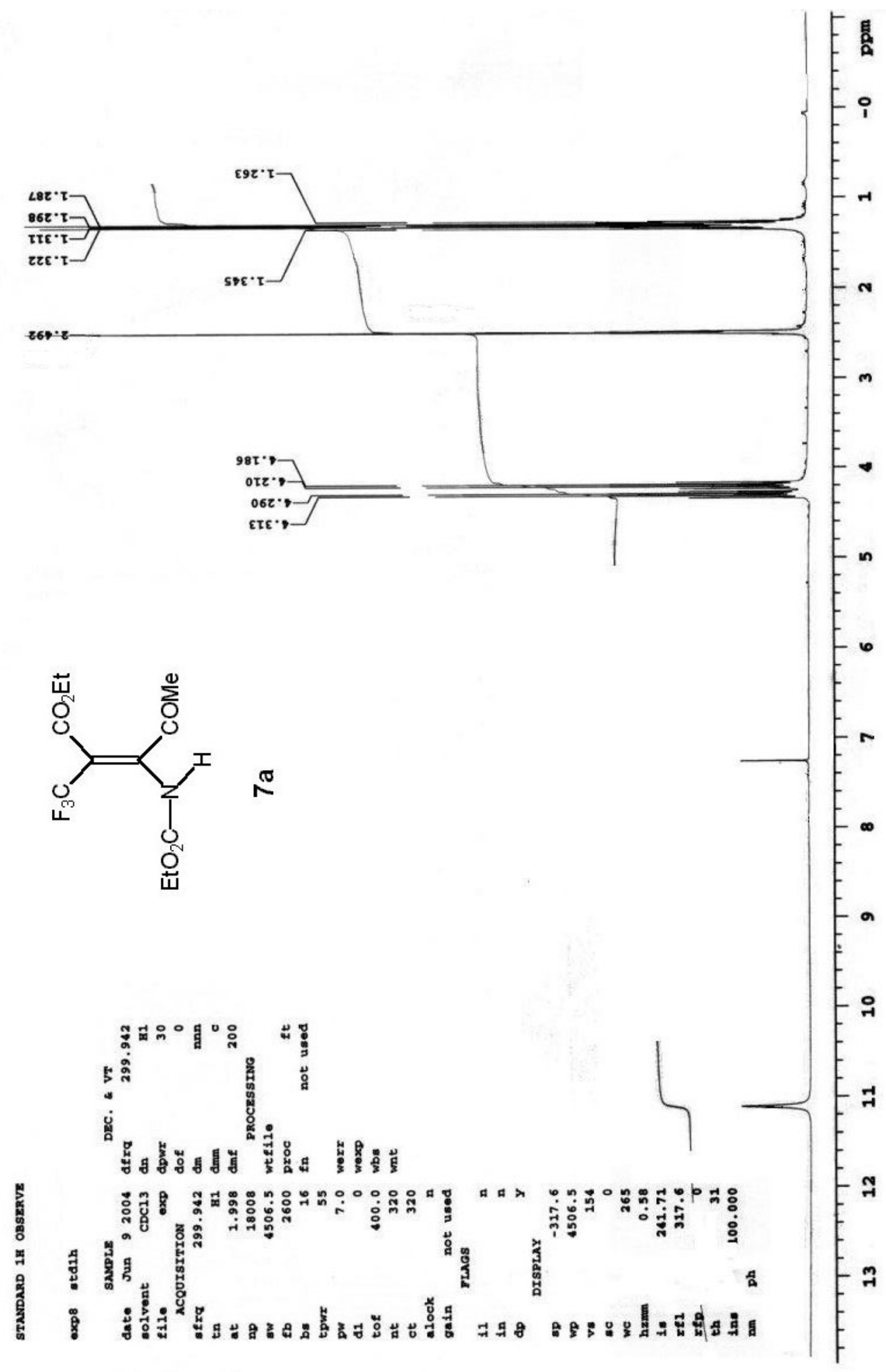




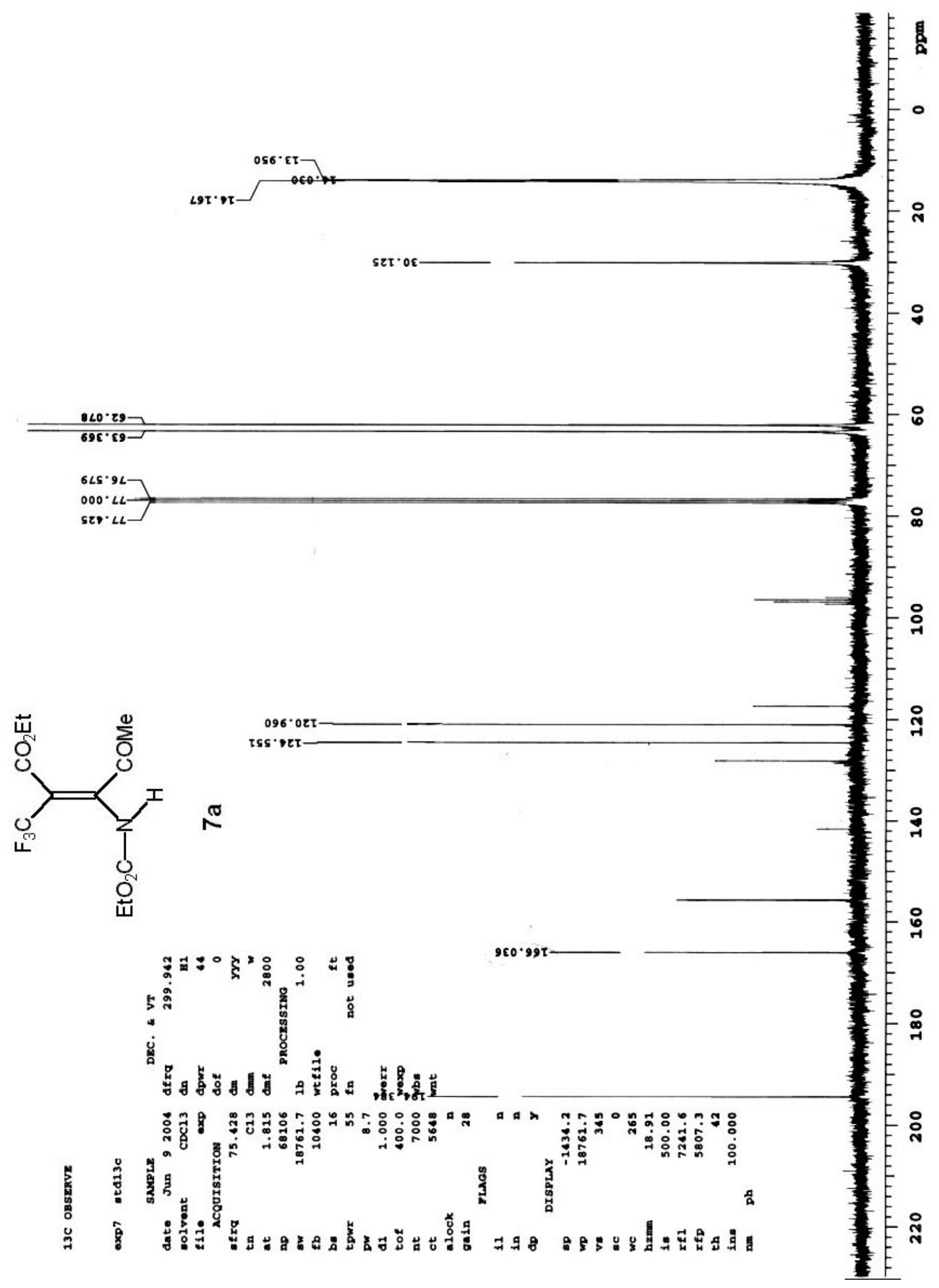




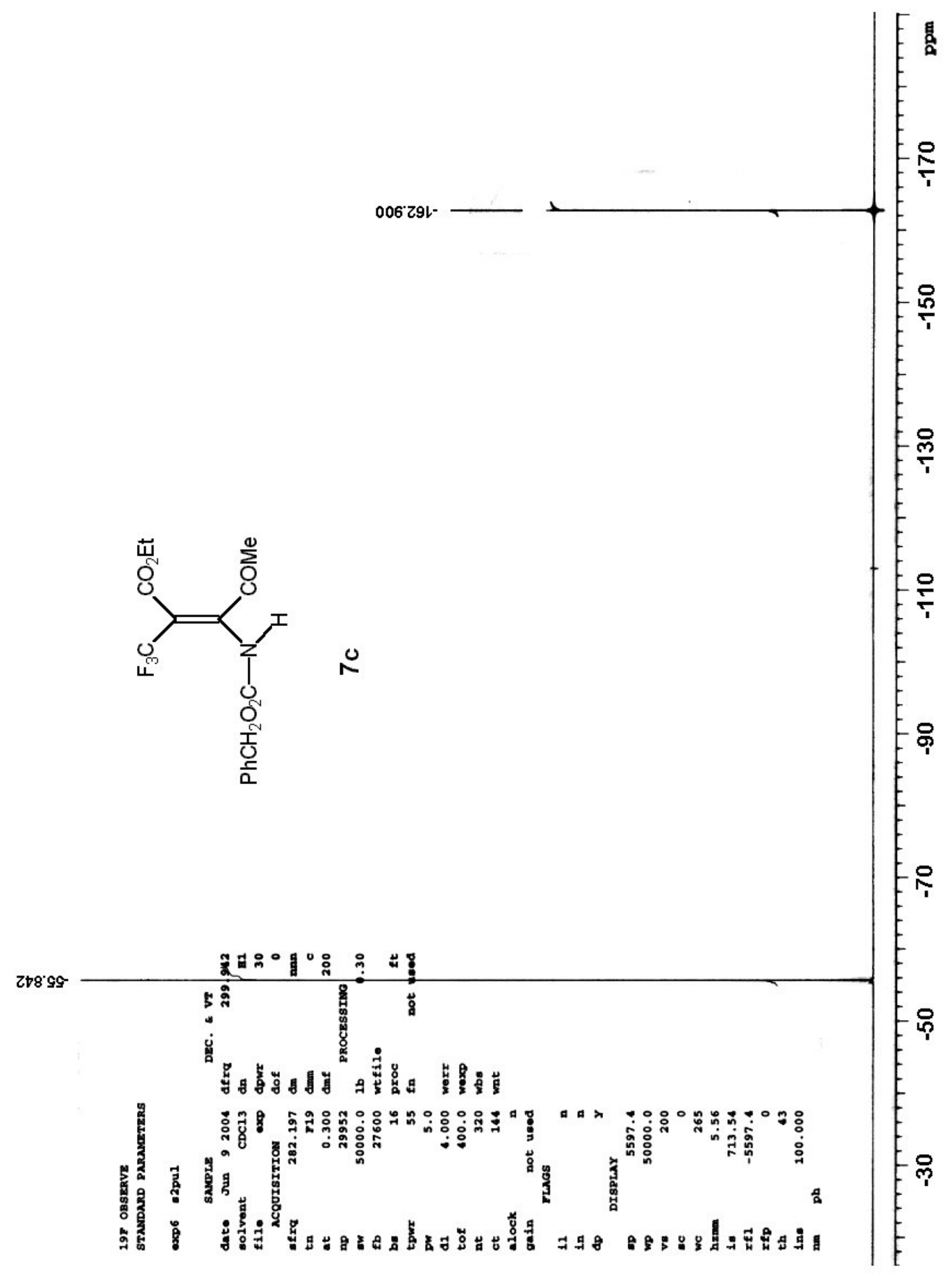




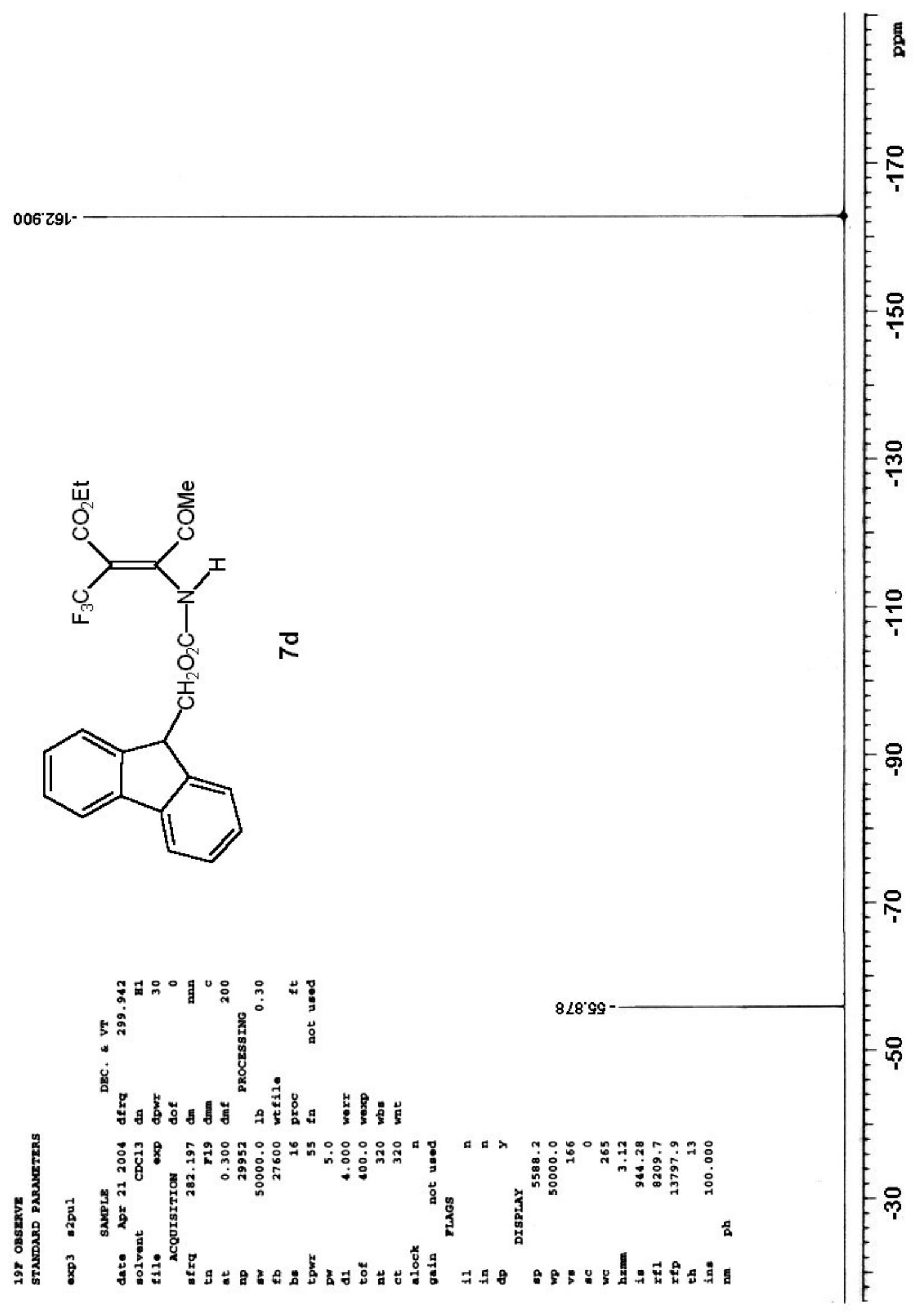




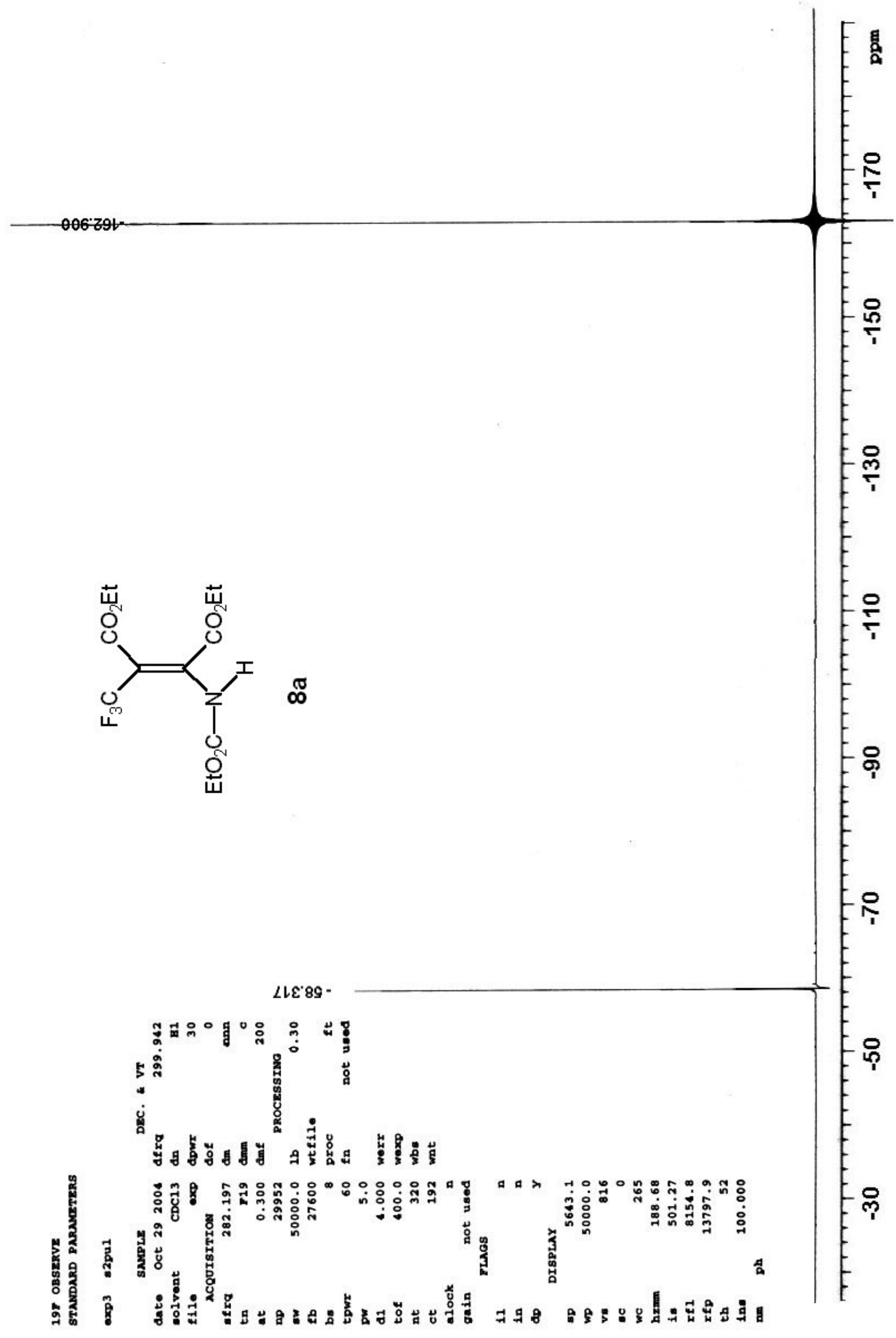




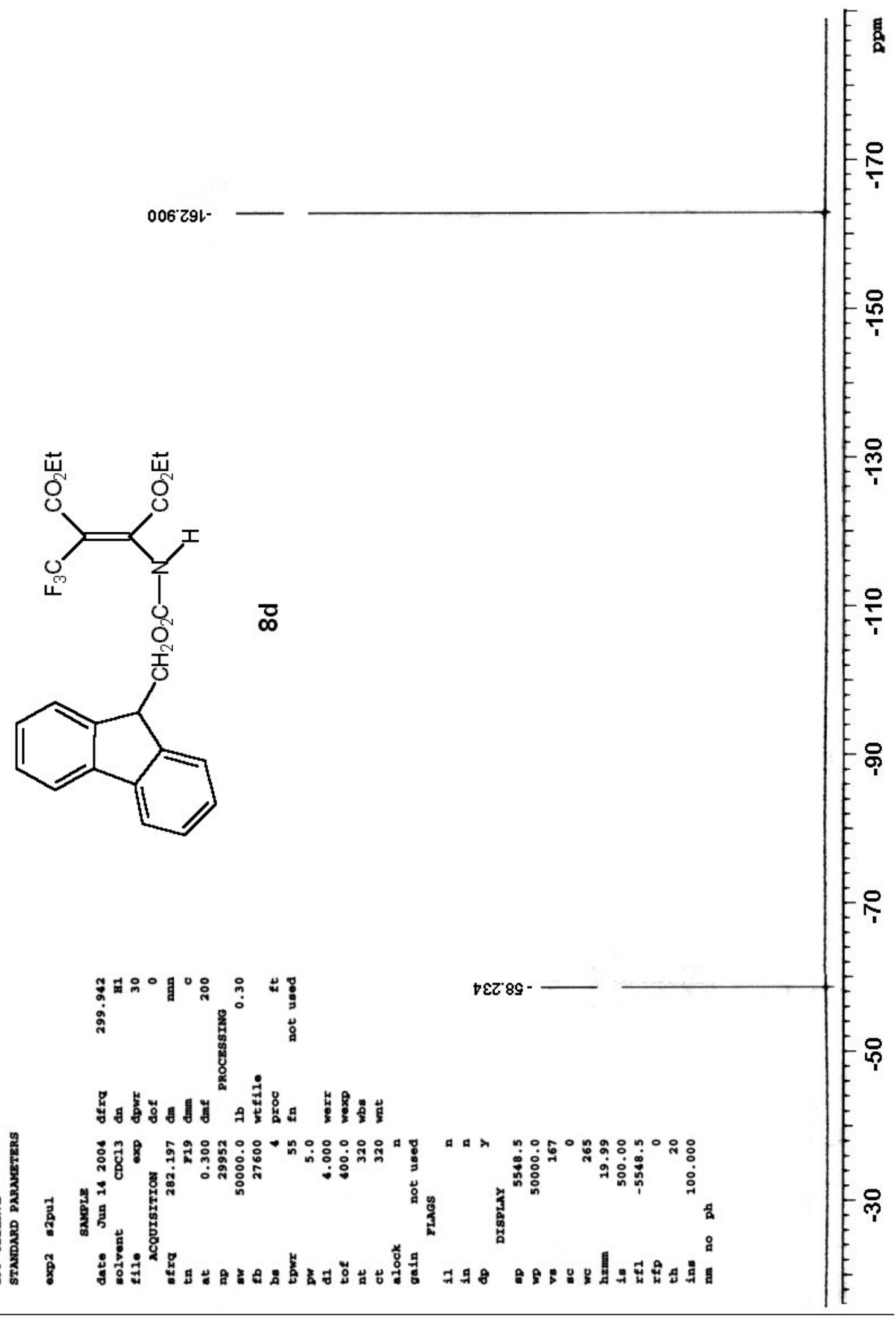




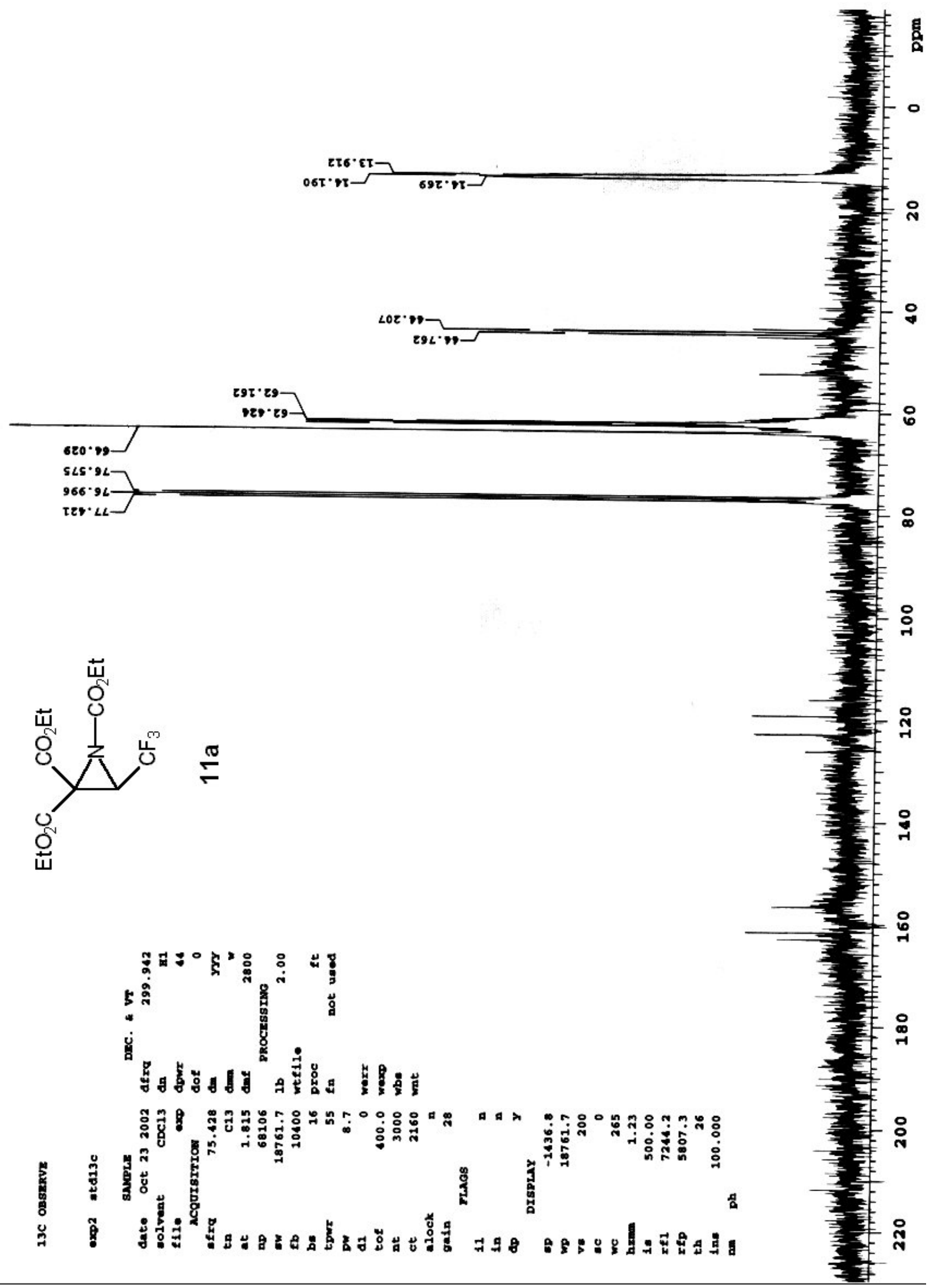




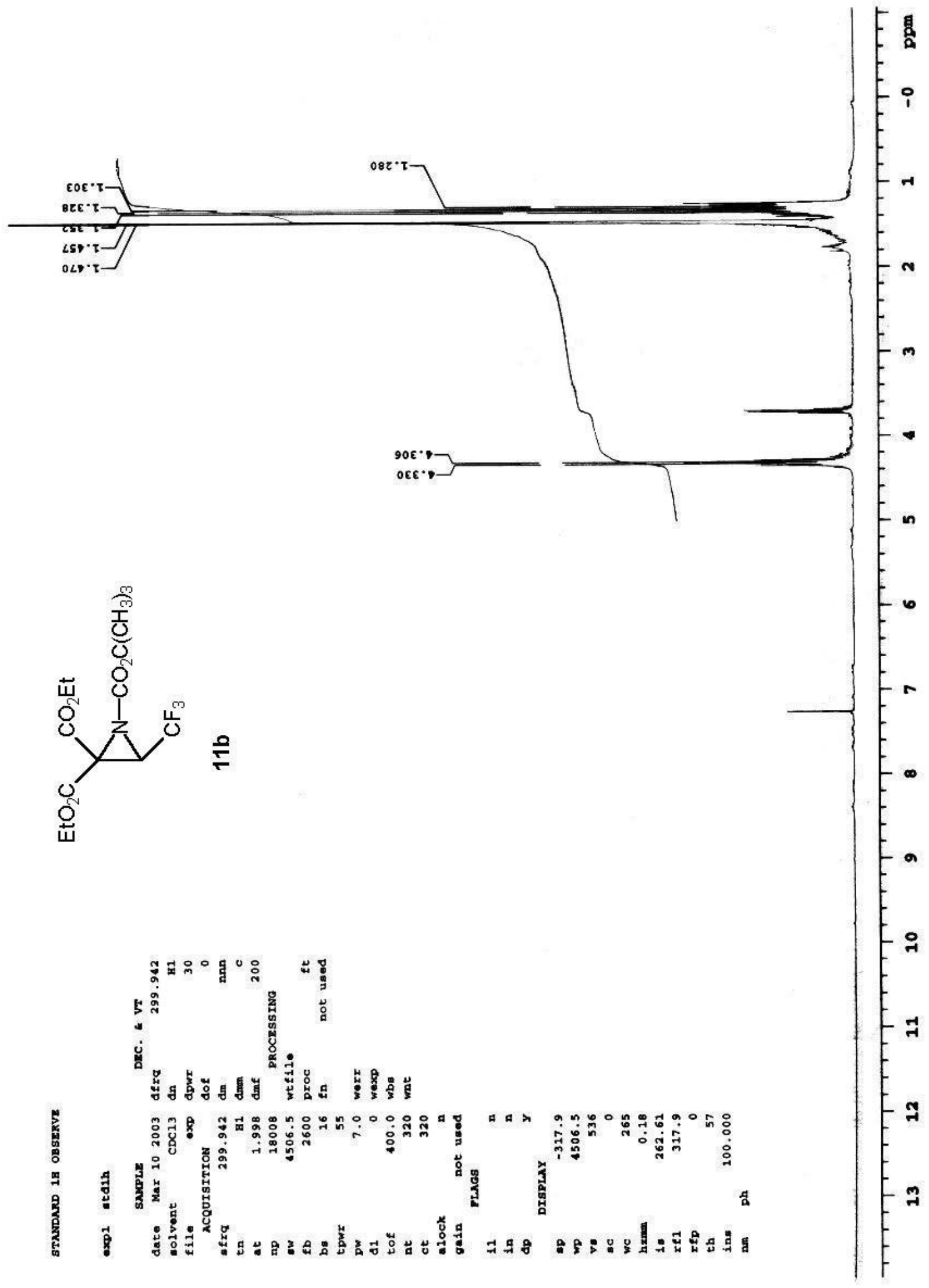




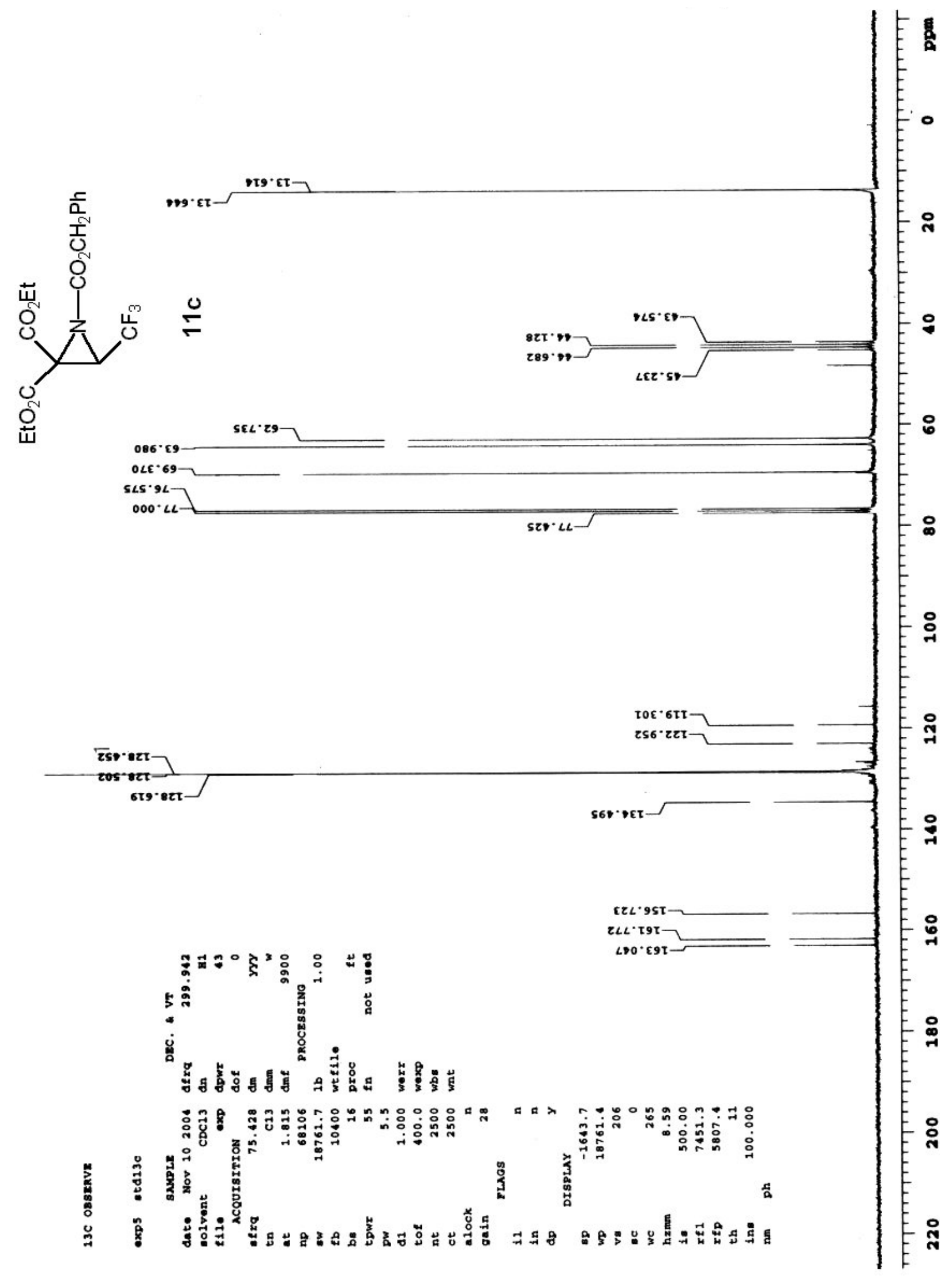




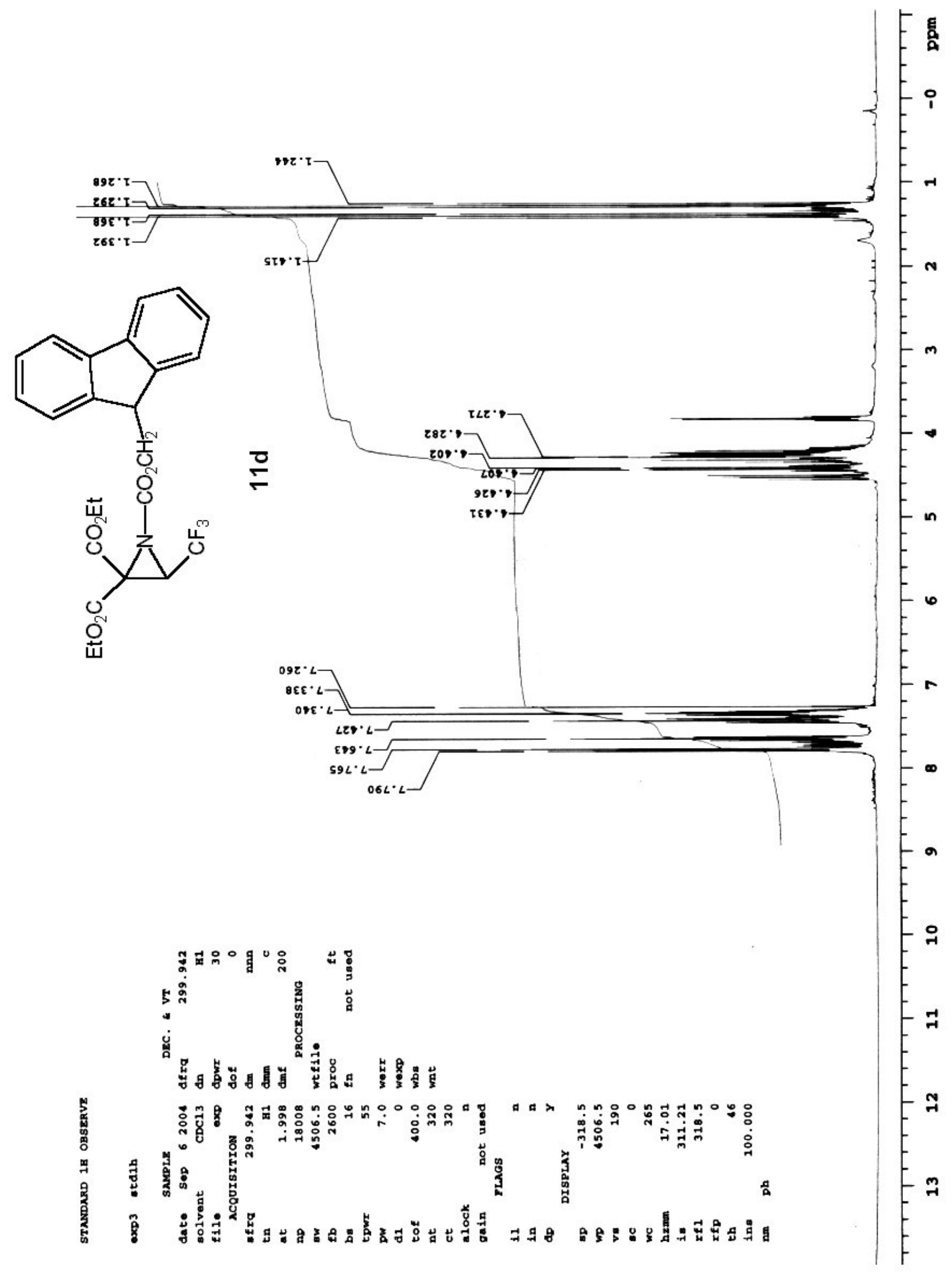


